\title{
- A Universal Approach to Coverage Probability and Throughput Analysis for Cellular Networks
}

\author{
Hui Zhang, Sheng Chen, Fellow, IEEE, Liang Feng, Yifeng Xie, and Lajos Hanzo, Fellow, IEEE
}

\begin{abstract}
4 Abstract-This paper proposes a novel tractable approach 5 for accurately analyzing both the coverage probability and the 6 achievable throughput of cellular networks. Specifically, we de7 rive a new procedure referred to as the equivalent uniform8 density plane-entity (EUDPE) method for evaluating the other-cell 9 interference. Furthermore, we demonstrate that our EUDPE 10 method provides a universal and effective means to carry out the 11 lower bound analysis of both the coverage probability and the 12 average throughput for various base-station distribution models 13 that can be found in practice, including the stochastic Poisson 14 point process (PPP) model, a uniformly and randomly distributed 15 model, and a deterministic grid-based model. The lower bounds 16 of coverage probability and average throughput calculated by our 17 proposed method agree with the simulated coverage probability 18 and average throughput results and those obtained by the existing 19 PPP-based analysis, if not better. Moreover, based on our new 20 definition of cell edge boundary, we show that the cellular topology 21 with randomly distributed base stations (BSs) only tends toward 22 the Voronoi tessellation when the path-loss exponent is suffi23 ciently high, which reveals the limitation of this popular network 24 topology.
\end{abstract}

25 Index Terms-Achievable throughput, cellular coverage, cellu26 lar networks, deterministic grid-based model, Poisson point pro27 cess (PPP) model, uniformly and randomly distributed model.

\section{INTRODUCTION}

30 INCE cellular systems are under growing pressure to in$31 \mathrm{~N}$ crease the volume of data delivered to consumers, es32 tablishing an accurate performance prediction model is of 33 prime significance [1]. Cellular systems are evolving into a 34 large-scale heterogeneous network architecture, constructed by 35 overlapping network tiers, such as macrocells, picocells, fem36 tocells, etc. [2]-[4]. The traditional cellular analysis relying on 37 an idealized hexagonal model does not realistically represent 38 the actual distribution of cells. Clearly, such a simplistic model

Manuscript received March 5, 2014; revised August 31, 2014; accepted October 28, 2014. This work was supported in part by the National Natural Science Foundation Project of China under Grant 61101084 and in part by the Fundamental Research Funds for the Central Universities, China. The review of this paper was coordinated by Dr. Y. Ma.

H. Zhang, L. Feng, and Y. Xie are with the Wireless Communications Laboratory, Information College, Nankai University, Tianjin 300071, China (e-mail: zhangh@nankai.edu.cn; fengliang201203@gmail.com; xyfhope@ gmail.com).

S. Chen is with the School of Electronics and Computer Science, University of Southampton, Southampton SO17 1BJ, U.K., and also with King Abdulaziz University, Jeddah 21589, Saudi Arabia (e-mail: sqc@ecs.soton.ac.uk).

L. Hanzo is with School of Electronics and Computer Science, University of Southampton, Southampton SO17 1BJ, U.K. (e-mail: 1h@ecs.soton.ac.uk).

Color versions of one or more of the figures in this paper are available online at http://ieeexplore.iee.org.

Digital Object Identifier 10.1109/TVT.2014.2366597 cannot be used for accurately modeling real-world cellular 39 networks and for analyzing the coverage probability and the 40 achievable throughput. Two mathematical models, i.e., the cel- 41 lular system interference model and the base station (BS) or cell 42 distribution model, are fundamental in the coverage analysis.

\section{A. Related Work and Motivation}

According to [5], the interference models can generally be di- 45 vided into two types: empirical models and statistical-physical 46 models. The construction of an empirical interference model 47 relies on first measuring the interference and then fitting a 48 mathematical model to the data. By contrast, the derivation of 49 a statistical-physical model usually relies on the mathematical 50 modeling of the interference. The classic Wyner model [6] was 51 proposed in 1994, and since then, it has been widely adopted in 52 the analysis of cellular networks. This model assumes that the 53 interference is constituted by the sum of the signals transmitted 54 from the adjacent cells (typically only considering two neigh- 55 bors), which is often multiplied by a fixed scaling factor or gain 56 to represent the specific intensity of the interferers [6]-[9]. 57

Determining the most beneficial positions of the BSs rep- 58 resents a critical planning problem in cellular networks. 59 Traditional methods usually place the BSs deterministically on 60 a regular grid, despite the fact that, in practice, the positions 61 of BSs are influenced by many random factors. Taking into 62 account the randomness of BS locations, in [10] and [11], a 63 stochastic-geometry-based method for modeling the positions 64 of the BSs was derived, whereas in [12] and [13], it was 65 proposed that the BSs be placed according to a homogeneous 66 Poisson point process (PPP) associated with a given intensity 67 [12], [13]. However, since the cellular network is gradually 68 evolving into a large-scale heterogeneous network associated 69 with multiple-tier random BS locations, the design challenge 70 becomes more grave. A recent contribution [14] has demon- 71 strated that the BS locations may be drawn from a PPP, partic- 72 ularly for single-tier networks. In [5], the statistical-physical 73 modeling of cochannel interference (CCI) was investigated 74 by assuming that the geographic distribution of interferers is 75 known a priori and that the interferers belong to a Poisson 76 field, with each individual interferer having a random session 77 life time. In [15], a mathematical theory based on a spatially 78 homogeneous PPP was provided to analyze the effects of 79 interference, which models the spatial distribution of the nodes 80 over the 2-D infinite plane by PPP theory.

The PPP model was used in [12] for establishing a hetero- 82 geneous network model of a single-tier macrocell network. 83 
84 Based on this PPP model, the calculation of the cumulative 85 interference imposed by all surrounding BSs can be carried 86 out with the aid of the Laplace transform and the probability 87 generating function [12], [14]. Furthermore, the coverage prob88 ability expression was deduced for the specific scenario, when 89 the interference experiences Rayleigh fading, and the results 90 of [12] and [14] demonstrated that the analysis based on the 91 PPP-aided modeling represent the lower bound of simulation 92 results. ${ }^{1}$ Similarly, the achievable average rate was also calcu93 lated. Although the PPP model is adopted for the analysis of 94 cellular networks, it is only accurate for sparse networks. By 95 contrast, it suffers from a lack of realism in the case of dense 96 networks since it may place several BSs far too closely together, 97 which does not make practical sense as such a situation will not 98 occur in a real BS deployment. It may impose excessive CCI if 99 too many BSs are deployed too densely. Noting this weakness 100 of the PPP model, some balanced measures are suggested to 101 alleviate this drawback in [12], but this weakness cannot be fun102 damentally eliminated by these measures. Moreover, the PPP103 based analysis relies on the assumption that the transmitters are 104 independently distributed [16].

105 A range of alternative stochastic-geometry-based methods 106 have also been used in the analysis of wireless networks [17], 107 [18]. For example, in [17], the Matérn hard-core process was 108 invoked for modeling the classic carrier sense multiple access 109 (CSMA) protocol and for analyzing its throughput, where the 110 presence of interferers within a given radius around any trans111 mitter was prevented. The Matérn point process [19] was modi112 fied in [18] to model the CSMA with collision avoidance, which 113 yields more realistic results by applying the aforementioned 114 interference-exclusion zone around all possible transmitters. 115 However, coverage analysis based on a Matérn hard-core pro116 cess is difficult to carry out [20] since the probability generating 117 functional of a Matérn hard-core process does not exist. It was 118 argued in [20]-[22] that only the Matérn type II process causes 119 a level of interference comparable to that predicted by a PPP 120 and, therefore, for interference-based performance analysis, the 121 Matérn type II process may be safely approximated by the 122 corresponding nonhomogeneous PPP [20]-[22].

\section{B. Our Approach and Contributions}

124 Against the above background, we propose a novel universal 125 approach for tractable and accurate coverage analysis of cellu126 lar networks. Our contributions are as follows.

127 1) Physical Analysis of Hexagonal/Voronoi Cells: To inter128 pret the various geometric-based cellular models from a physi129 cal perspective, we provide a tangible generic definition of the 130 cell edge boundary for our theoretical analysis, where the cell 131 boundary is directly linked to the path-loss exponent. Specif132 ically, we show that the traditional hexagonal topology natu133 rally emerges from the grid-based model, given a sufficiently 134 high path-loss exponent, whereas the Voronoi tessellation nat135 urally emerges from the random BS distribution model, again

\footnotetext{
${ }^{1}$ The simulation results are referred to as "experimental" or "actual" in [12], which is inappropriate.
}

provided that the path-loss exponent is sufficiently high. How- 136 ever, such a high path-loss exponent is unrealistic in real trans- 137 mission environments. Therefore, our physical analysis reveals 138 the fundamental limitation of these purely graphic-based cellu- 139 lar topologies, namely, lack of the connection to the underlying 140 signal transmission medium. In fact, we demonstrate that the 141 cell edge boundary shows irregular near-circular shapes, given a 142 more realistic path-loss exponent of around 3, which cannot be 143 modeled accurately by either hexagonal or Voronoi tessellation. 144

2) EUDPE-Based Other-Cell Interference Model: We pro- 145 pose a universal model for evaluating the other-cell interfer- 146 ence, which we refer to as the equivalent uniform-density 147 plane-entity (EUDPE) method. This generic EUDPE model can 148 be used to calculate the cumulative other-cell interference for 149 all the existing BS distribution models that can be found in 150 practice, including both stochastic and deterministic cellular 151 network models, such as the stochastic Poisson distributed (PD) 152 and uniformly distributed (UD) BS models and the determinis- 153 tic grid-based BS model.

3) Lower Bound Analysis for Coverage Probability and 155 Average Achievable Rate: Based on the proposed generic 156 EUDPE interference model, we perform the low-bound anal- 157 ysis of both the coverage probability and the average achiev- 158 able rate for various BS distribution models, specifically, the 159 stochastic PD and UD BS models and the deterministic grid- 160 based BS model, which may be viewed as a degenerated or spe- 161 cial case of the UD BS model. For realistic path-loss exponents, 162 the coverage probability and average achievable throughput 163 results provided by our proposed analysis approach agree with 164 the simulated coverage probability and achievable throughput. 165 In fact, their match is as good or better than that of the PPP- 166 based analysis. The results also show that the noise only has a 167 modest effect on the coverage probability and achievable rate. 168

The remainder of this paper is organized as follows. In 169 Section II, the downlink cellular system model is briefly in- 170 troduced, which is followed by our new physical analysis of 171 cell edge boundary. Section III is devoted to the derivation of 172 our EUDPE-based interference model. The low-bound analysis 173 of the coverage probability based on the EUDPE method is 174 deduced in Section IV for both stochastic BS distribution 175 models and deterministic grid-based BS models, whereas the 176 corresponding low-bound analysis is presented in Section V. 177 Our conclusions are offered in Section VI.

\section{Downlink Cellular System Model}

Throughout our discussions, the index set of the BSs, which 180 are deployed according to some distribution, is denoted by $\Phi, 181$ whereas the serving BS's index is denoted by $b_{0}$. Furthermore, 182 the average density of BSs is $\rho$. Let $P$ be the transmitted power 183 of a BS, $R$ be the serving BS's coverage radius, $R_{\mathrm{nw}}$ be the 184 distance from the serving BS to the edge of the network, and 185 $r_{i}$ denotes the distance from the $i$ th BS to the user equipment 186 (UE) concerned. If we denote the average coverage area of a 187 BS by $\mathbb{E}\left[A_{s}\right]$ with $\mathbb{E}[]$ representing the expectation operator, 188 then $\mathbb{E}\left[A_{s}\right]=1 / \rho$. We will also use $2 R$ to denote the average 189 distance between two neighboring BSs, and we have $R \propto 190$ $\sqrt{\mathbb{E}\left[A_{s}\right]}$. 


\section{A. SINR Model}

193 The wireless channel linking the $i$ th BS and the UE con194 cerned is modeled by a complex-valued channel tap that takes 195 into account the path loss with a path-loss exponent of $\alpha$, the 196 fast Rayleigh fading coefficient with an instantaneous power 197 or a squared magnitude of $h_{i}$, and the channel's additive white 198 Gaussian noise (AWGN) with noise power of $\sigma^{2}$. The average 199 of the random variable $h_{i}$ is denoted by $\bar{h}$; therefore, $h_{i}$ follows 200 the exponential distribution with the mean $\bar{h}$.

201 Let us assume that the intracell UE-to-UE interference is neg202 ligible. Then, the signal-to-interference-plus-noise ratio (SINR) 203 experienced at this UE can be expressed as follows:

$$
\mathrm{SINR}=\frac{P h_{0} r_{0}^{-\alpha}}{I_{r}+\sigma^{2}}
$$

204 where the interference arriving from all the interfering cells is 205 given by

$$
I_{r}=\sum_{i \in \Phi \backslash b_{0}} P h_{i} r_{i}^{-\alpha}
$$

206 If the target SINR value is $T$, then the actual SINR must obey 207 SINR $>T$, which requires

$$
h_{0}>P^{-1} \operatorname{Tr}_{0}^{\alpha}\left(\sigma^{2}+I_{r}\right) .
$$

208 Thus, the probability distribution of $h_{0}$ should be taken into 209 account in the analysis of both the coverage probability and the 210 average rate. Furthermore, intuitively, the given SINR model 211 determines the coverage area of each BS; therefore, it influences 212 the cell shape or boundary.

\section{B. Physical Analysis of Cell Edge Boundary}

214 As aforementioned, the grid-based cellular model is conve215 nient but is too idealistic. By contrast, the Voronoi tessellation is 216 considered to match the random BS deployment in relative flat 217 urban areas reasonably well [12], [23]. Hence, cellular networks 218 can be analyzed using Voronoi diagram theory, albeit this has 219 not been explained with the aid of a physically tangible per220 spective. More specifically, both the energy efficiency and cov221 erage of cellular networks may be analyzed based on Voronoi 222 tessellation [24], [25]. Fig. 1 shows a random distribution of 223 the BSs with the cell boundaries corresponding to a Voronoi 224 tessellation. Note that in both the grid-based and Voronoi-based 225 cellular topologies, the cell boundaries are determined purely 226 by the geometric property of the BS distribution, and they are 227 completely independent of the actual physical interference that 228 the network is experiencing.

229 To interpret the cell edge boundary from a physical percep230 tively, namely, linking it better to the underlying physics of 231 signal transmission medium, let us now introduce the following 232 definition that formally defines the cell edge boundary.

233 Definition 1: The cell edge boundary is constituted by the 234 group of points where the strength of the desired signal received 235 from the serving BS equals to the interfering signal's strength. 236 In other words, at the cell edge boundary, the desired signal237 to-interference ratio (SIR) is equal to 1 . This definition of cell

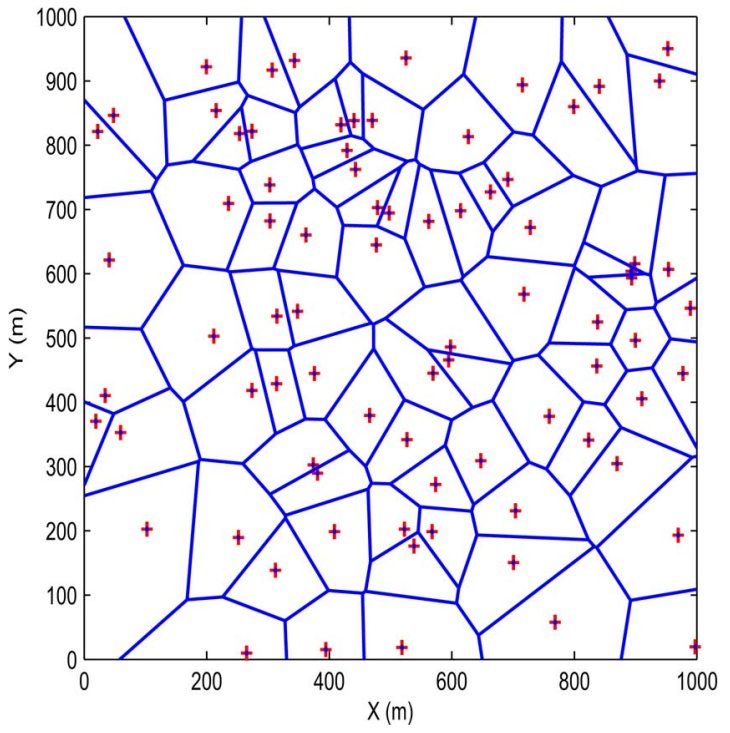

Fig. 1. Random distribution of the BSs marked by + , with the cell boundaries corresponding to a Voronoi tessellation.

edge boundary is both intuitive and practical since, within the 238 coverage area of a BS, the desired signal should be stronger than 239 the interfering signal, yielding SIR $>1$. Let us denote the $i$ th 240 BS location as the point $z_{i}$, where $i \in \Phi$. Furthermore, denote 241 the distance from $z_{i}$ to a point $z$ as $\left|z-z_{i}\right|$. The desired signal 242 power at the point $z$ provided by the $i$ th $\mathrm{BS}$ is given by

$$
\mathrm{S}(z)=\mathbb{E}\left[P h_{i}\left|z-z_{i}\right|^{-\alpha}\right]=P \bar{h}\left|z-z_{i}\right|^{-\alpha}
$$

while the interfering signal's power at $z$ is given by

$$
\begin{aligned}
\mathrm{I}(z) & =\mathbb{E}\left[I_{r}(z)\right]=\mathbb{E}\left[\sum_{j \in \Phi \backslash i} P h_{j}\left|z-z_{j}\right|^{-\alpha}\right] \\
& =P \bar{h} \sum_{j \in \Phi \backslash i}\left|z-z_{j}\right|^{-\alpha} .
\end{aligned}
$$

Thus, with respect to the $i$ th BS, the SIR at the point $z$ is 245 given by

$$
\operatorname{SIR}(z)=\frac{\left|z-z_{i}\right|^{-\alpha}}{\sum_{j \in \Phi \backslash i}\left|z-z_{j}\right|^{-\alpha}} .
$$

Therefore, at the $i$ th cell's edge boundary, we have $\operatorname{SIR}(z)=1.247$

In Figs. 2 and 3, the distribution of the BSs is based on the 248 same regular grid network model, and the number of BSs is 33. 249 As shown in Fig. 2, the shape of each cell in the network is 250 approximately a regular circle given the path-loss exponent of 251 $\alpha=3$. By contrast, observe in Fig. 3 that the cell shape changes 252 into a hexagonal one when the path-loss exponent is increased 253 to $\alpha=10$.

In Figs. 4 and 5, the locations of the 33 BSs are randomly 255 drawn from the uniform distribution across the entire network 256 area. The cells now approximately have irregularly circular 257 shapes when the path-loss exponent is $\alpha=3$, but interestingly, 258 it is the Voronoi tessellation that naturally emerges when the 259 path-loss exponent is increased to $\alpha=10$. 


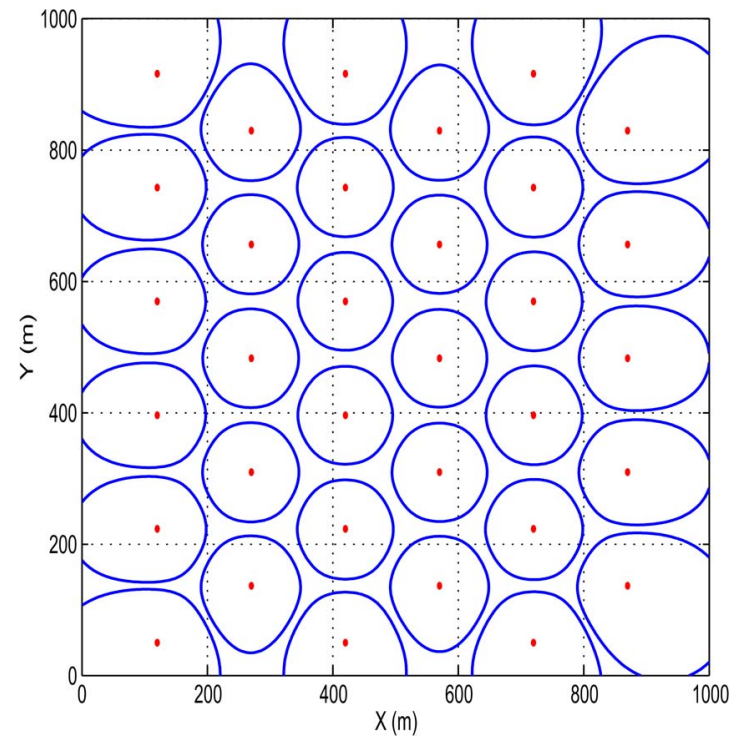

Fig. 2. Cell edge boundaries of the grid network model with the 33 BS locations marked by dots, as determined by $\operatorname{SIR}(z)=1$. The path-loss exponent is $\alpha=3$.

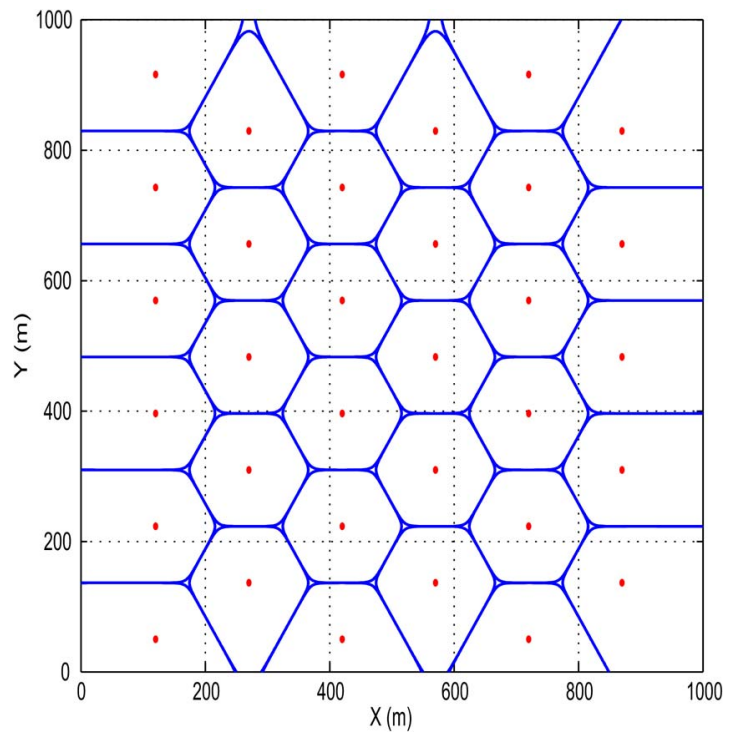

Fig. 3. Cell edge boundaries of the grid network model with the 33 BS locations marked by dots, as determined by $\operatorname{SIR}(z)=1$. The path-loss exponent is $\alpha=10$.

261 The given results demonstrate that our Definition 1 of cell 262 edge boundary is a physically plausible one for analyzing the 263 network, and both the hexagonal topology and the Voronoi 264 tessellation naturally emerge according to this definition, de265 pending on whether the geographic distribution of BSs is deter266 ministic or random and providing that the path-loss exponent 267 is sufficiently high. Note that such a high path-loss exponent 268 is unrealistic in real transmission environments. Therefore, 269 our analysis of cell edge boundary reveals a weakness of the 270 popular hexagonal and Voronoi network topologies, namely, 271 they do not reflect the underlying signal transmission medium. 272 Significantly, given a more realistic path-loss exponent of ap273 proximately three, the cell edge boundary exhibits irregular

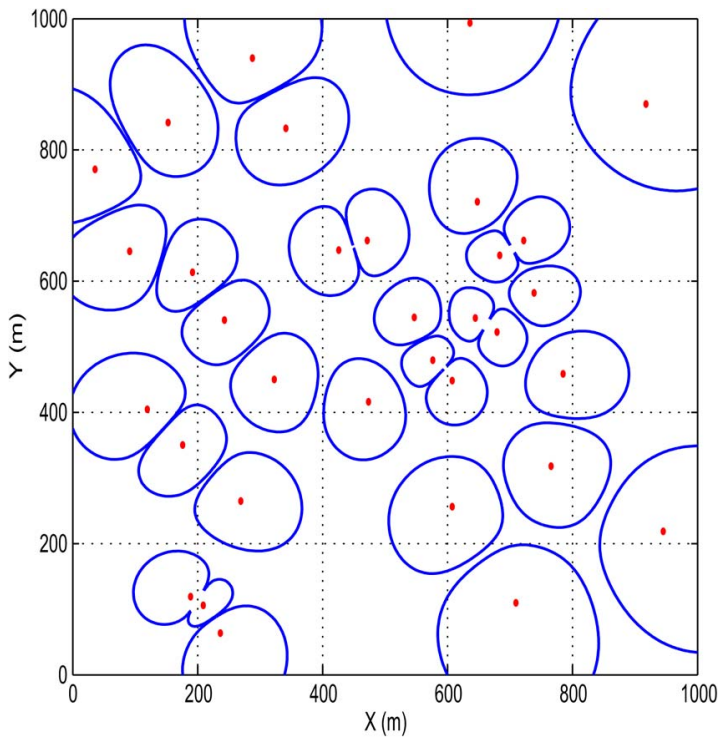

Fig. 4. Cell edge boundaries of the randomly distributed network model with the 33 BS locations marked by dots, as determined by $\operatorname{SIR}(z)=1$. The pathloss exponent is $\alpha=3$.

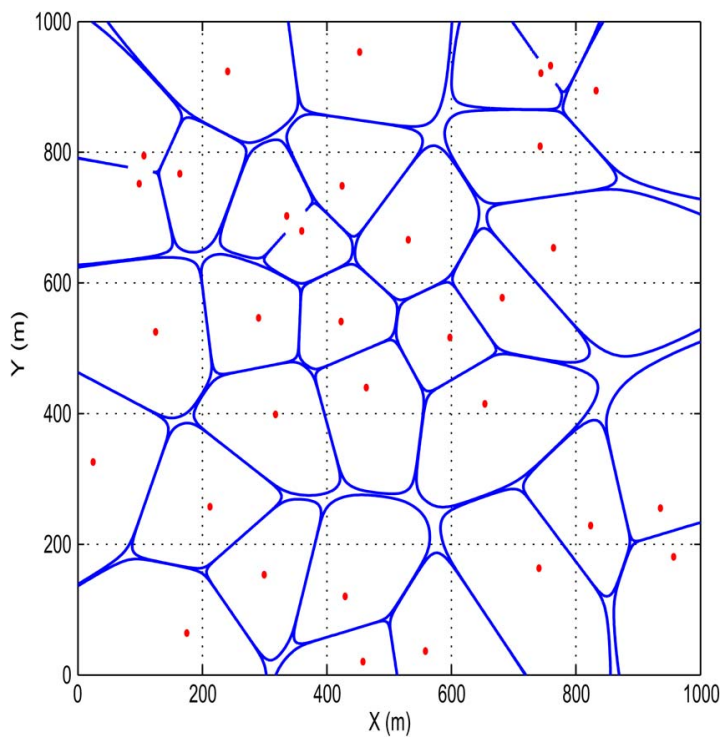

Fig. 5. Cell edge boundaries of the randomly distributed network model with the 33 BS locations marked by dots, as determined by $\operatorname{SIR}(z)=1$. The pathloss exponent is $\alpha=10$.

near-circular cell shapes, for which neither hexagonal topology 274 nor Voronoi tessellation can be used to accurately model. 275 Furthermore, the "weak" coverage areas that are left outside 276 any cell boundary, where the desired signal is weaker than the 277 interfering signals, as shown in Fig. 4, highlight the benefits of 278 employing collaborative relaying techniques.

\section{Equivalent Uniform Density Plane-Entity FoR 280 CUMUlative INTERFERENCE CALCULATION

To accurately analyze the coverage probability and the 282 achievable rate, it is necessary to find an efficient means for 283 


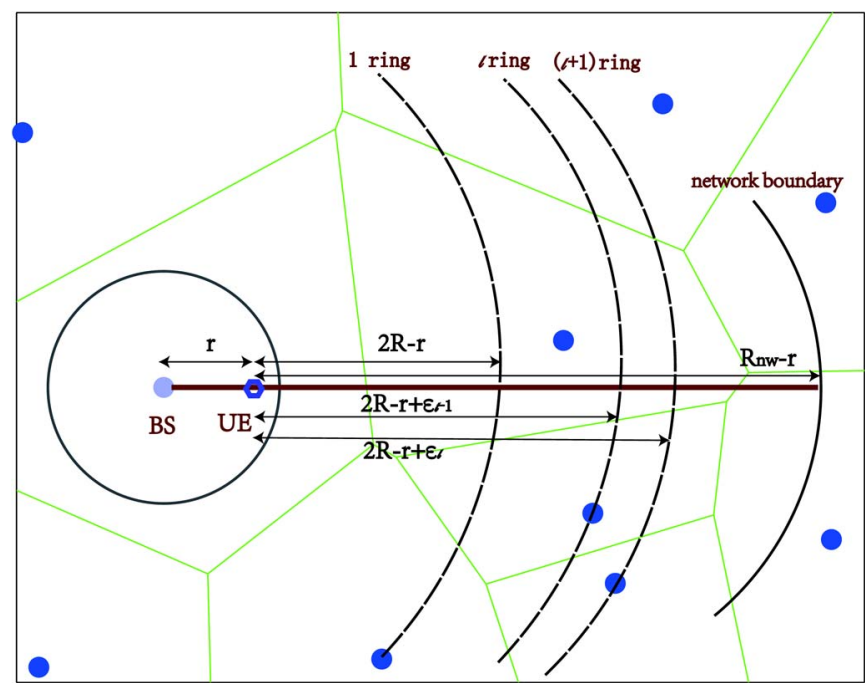

Fig. 6. Proposed EUDPE method for calculating the other-cell interference.

284 cumulative interference calculation. By considering the dis285 tribution of the interference imposed by the BSs in the law 286 of large numbers and combining it with the fluid model of 287 [26], we propose the EUDPE method for calculating the cu288 mulative interference. The basic idea of this EDUPE method 289 is as follows. Although the actual geographic distribution of 290 BSs always shows a certain degree of irregularity, we may 291 define a group of equivalent and uniformly distributed BSs for 292 approximating the other-cell CCI. Since, in real-world cellular 293 networks, the actual geographic distribution of BSs is often 294 close to a uniform random distribution, such an approximation 295 is sufficiently accurate. It is worth emphasizing however that 296 we do not assume a uniform and random BS distribution for 297 the actual network to be modeled. More specifically, given 298 a network having the average BS density of $\rho$, we approx299 imate this network with an equivalent network whose BSs 300 are uniformly distributed and whose BS density is also $\rho$. 301 Such a network is termed the equivalent EUDPE of the given 302 network. With the aid of our EUDPE method, we can calcu303 late or approximate the cumulative interference for any given 304 network.

305 Fig. 6 illustrates the concept of the EUDPE, where the 306 serving BS is assumed the origin of the polar coordinate plane. 307 Since the coverage radius of a BS is $R$, the distance between 308 two neighboring BSs is $2 R$, where $R \propto(1 / \sqrt{\rho})$. For notational 309 simplification, we drop the subscript 0 from $r_{0}$ and denote the 310 distance from the serving BS to the UE as $r$, where $0 \leq r \leq R$. 311 Thus, the distance from the nearest interfering BS to the UE 312 is $(2 R-r)$. As shown in Fig. 6, the network's coverage area 313 is partitioned by the $N_{r}$ rings, and the distance from the UE 314 to the $l$ th ring is given by $\left(2 R-r+\varepsilon_{l-1}\right)$, where $1 \leq l \leq$ $315 N_{r}$ with $\varepsilon_{0}=0$ and $\left(2 R-r+\varepsilon_{N_{r}}\right)=R_{\mathrm{nw}}-r$. The number 316 of BSs within the area between the $l$ th and $(l+1)$ th rings 317 is approximately $\int_{0}^{2 \pi} \int_{2 R-r+\varepsilon_{l-1}}^{2 R-r+\varepsilon_{l}} \rho z d z d \theta$ when assuming the 318 equivalent EUDPE having the BS density of $\rho$. Furthermore, 319 each of these equivalent BSs has the same instantaneous 320 fast fading channel power of $\widetilde{h}_{l}$, and the mean of $\widetilde{h}_{l}$ is $\bar{h}$.
Thus, the cumulative interference $I_{r}$ can be approximated 321 according to

$$
\begin{aligned}
I_{r} & =\sum_{l=1}^{N_{r}} \int_{0}^{2 \pi} \int_{2 R-r+\varepsilon_{l-1}}^{2 R-r+\varepsilon_{l}} P \widetilde{h}_{l} z^{-\alpha} \rho z d z d \theta \\
& =\sum_{l=1}^{N_{r}} \frac{2 \pi \rho P \widetilde{h}_{l}}{\alpha-2}\left(\left(2 R-r+\varepsilon_{l-1}\right)^{2-\alpha}-\left(2 R-r+\varepsilon_{l}\right)^{2-\alpha}\right) .
\end{aligned}
$$

Theorem 1: The average of $I_{r}$ is given by

$$
\mathbb{E}\left[I_{r}\right]=\frac{2 \pi \rho P \bar{h}}{\alpha-2}\left((2 R-r)^{2-\alpha}-\left(R_{\mathrm{nw}}-r\right)^{2-\alpha}\right) .
$$

Proof: According to the Campbell-Mecke theorem [27], 324 we have

$$
\begin{aligned}
\mathbb{E} & {\left[\sum_{l=1}^{N_{r}} \frac{2 \pi \rho P \widetilde{h}_{l}}{\alpha-2}\left(\left(2 R-r+\varepsilon_{l-1}\right)^{2-\alpha}-\left(2 R-r+\varepsilon_{l}\right)^{2-\alpha}\right)\right] } \\
& =\sum_{l=1}^{N_{r}} \frac{2 \pi \rho P \mathbb{E}\left[\widetilde{h}_{l}\right]}{\alpha-2}\left(\left(2 R-r+\varepsilon_{l-1}\right)^{2-\alpha}-\left(2 R-r+\varepsilon_{l}\right)^{2-\alpha}\right) \\
& =\frac{2 \pi \rho P \bar{h}}{\alpha-2}\left((2 R-r)^{2-\alpha}-\left(R_{\mathrm{nw}}-r\right)^{2-\alpha}\right) .
\end{aligned}
$$

Typically, the path-loss exponent is $\alpha>2$ in realistic net- 327 works. Noting that $\left(R_{\mathrm{nw}}-r\right)^{2-\alpha} \rightarrow 0$ as $R_{\mathrm{nw}} \rightarrow+\infty$, we 328 have the following corollary.

Corollary 1: Given that the network's boundary is suffi- 330 ciently far away, namely, $R_{\mathrm{nw}} \rightarrow+\infty$, we have

$$
\mathbb{E}\left[I_{r}\right]=\frac{2 \pi \rho P \bar{h}}{\alpha-2}(2 R-r)^{2-\alpha} .
$$

\section{Coverage Probability Analysis Using

As mentioned earlier, the cellular system interference model 334 and the BS geographic distribution model are required in cov- 335 erage analysis. Our proposed EUDPE is a universal method 336 for evaluating the other-cell interference for all existing BS 337 distribution models, such as the stochastic PD and UD BS 338 models and the deterministic grid-based model.

\section{A. Coverage Probability Analysis Using EUDPE-PD}

Since a popular geographic BS distribution is the Poisson 341 distribution [12]-[15], we first consider the PD BS model. The 342 probability density function (pdf) of the Poisson distribution 343 can be derived using the method of [28]. Let $\lambda$ be the intensity 344 of the Poisson distribution that models the BS geographic 345 distribution and $R$ be the average coverage radius of a cell. 346 Then, the probability of having no BS that is closer than $x$ is 347 given by

$$
\mathbb{P}\{r>x\}=\mathbb{P}\{\text { No BS closer than } x\}=e^{-\lambda \pi x^{2}} .
$$


349 The corresponding cumulative distribution function (cdf) is 350 then given by

$$
\mathbb{P}\{r \leq x\}=F(x)=1-e^{-\lambda \pi x^{2}} .
$$

351 Therefore, the pdf is defined as

$$
f(r)=\frac{d F(r)}{d r}=2 \pi \lambda r e^{-\pi \lambda r^{2}} .
$$

352 Given the SINR threshold $T$, the intensity $\lambda$ and the path-loss 353 exponent $\alpha$, the coverage probability is defined as

$$
\begin{aligned}
p_{c}(T, \lambda, \alpha) & =\mathbb{E}_{r}\left[\mathbb{E}_{I_{r}}[\mathbb{P}\{\operatorname{SINR}>T\}]\right] \\
& =\int_{r>0} \mathbb{E}_{I_{r}}\left[\mathbb{P}\left\{h_{0}>P^{-1} \operatorname{Tr}^{\alpha}\left(\sigma^{2}+I_{r}\right)\right\}\right] 2 \pi \lambda r e^{-\pi \lambda r^{2}} d r
\end{aligned}
$$

354 where $\mathbb{E}_{r}[\bullet]$ denotes the expectation with respect to the random 355 variable $r$.

356 1) Lower Bound for the Probability of SINR Larger Than 357 Threshold: Noting that $h_{0}$ obeys the exponential distribution 358 with the mean $\bar{h}$, the probability of the SINR larger than the 359 threshold $T$ (averaged over the interference) is given by

$$
\begin{aligned}
\mathbb{E}_{I_{r}}\left[\mathbb{P}\left\{h_{0}>P^{-1} \operatorname{Tr}^{\alpha}\left(\sigma^{2}+I_{r}\right)\right\}\right] & \\
= & e^{-\bar{h} P^{-1} T r^{\alpha} \sigma^{2}} \mathbb{E}_{I_{r}}\left[e^{-\bar{h} P^{-1} T r^{\alpha} I_{r}}\right] .
\end{aligned}
$$

360 Theorem 2: A lower bound for the probability of the SINR 361 greater than the threshold $T$ is expressed as

$$
\mathbb{E}_{I_{r}}\left[\mathbb{P}\left\{h_{0}>P^{-1} \operatorname{Tr}^{\alpha}\left(\sigma^{2}+I_{r}\right)\right\}\right] \geq e^{-\bar{h} T r^{\alpha} \eta(\alpha, r)}
$$

362 where

$$
\eta(\alpha, r)=P^{-1} \sigma^{2}+\frac{2 \pi \rho \bar{h}}{\alpha-2}\left((2 R-r)^{2-\alpha}-\left(R_{\mathrm{nw}}-r\right)^{2-\alpha}\right) .
$$

363

Proof: According to Jensen's inequality [29], we have

$$
\mathbb{E}_{I_{r}}\left[e^{-\bar{h} P^{-1} T r^{\alpha} I_{r}}\right] \geq e^{-\bar{h} P^{-1} T r^{\alpha} \mathbb{E}\left[I_{r}\right]} .
$$

364 Substituting (18) into (15) and noting $\mathbb{E}\left[I_{r}\right]$ of (8) leads to (16) 365 with $\eta(\alpha, r)$ given in (17).

366 Corollary 2: Given that the network boundary is sufficiently 367 far away, namely, $R_{\text {nw }} \rightarrow+\infty$

$$
\mathbb{E}_{I_{r}}\left[\mathbb{P}\left\{h_{0}>P^{-1} \operatorname{Tr}^{\alpha}\left(\sigma^{2}+I_{r}\right)\right\}\right] \geq e^{-\bar{h} T r^{\alpha} \xi(\alpha, r)}
$$

368 where

$$
\xi(\alpha, r)=P^{-1} \sigma^{2}+\frac{2 \pi \rho \bar{h}}{\alpha-2}(2 R-r)^{2-\alpha} .
$$

369 2) Lower Bound for the Coverage Probability: A lower 370 bound for the coverage probability $p_{c}(T, \lambda, \alpha)$ is given by the 371 following theorem.
Theorem 3: For the network where the BS geographic 372 distribution obeys the Poisson distribution of intensity $\lambda, 373$ a lower bound for the coverage probability $p_{c}(T, \lambda, \alpha)$ is 374 given by

$$
p_{c l}(T, \lambda, \alpha)=\pi \lambda \int_{0}^{R^{2}} e^{-\bar{h} T v^{\alpha / 2} \psi(\alpha, v)-\pi \lambda v} d v
$$

where $R$ is the coverage radius of the serving $\mathrm{BS}$, and

$$
\begin{aligned}
\psi(\alpha, v)=P^{-1} \sigma^{2}+\frac{2 \pi \rho \bar{h}}{\alpha-2}( & \left(2 R-v^{1 / 2}\right)^{2-\alpha} \\
& \left.-\left(R_{\mathrm{nw}}-v^{1 / 2}\right)^{2-\alpha}\right) .
\end{aligned}
$$

Proof: From (14) and Theorem 2, as well as noting that 377 $r \leq R$, we have

$$
p_{c l}(T, \lambda, \alpha)=\int_{0}^{R} 2 \pi \lambda r e^{-\bar{h} T r^{\alpha} \eta(\alpha, r)-\pi \lambda r^{2}} d r .
$$

By defining $r^{2}=v,(23)$ is transformed into (21) with $\psi(\alpha, v) 379$ given in (22).

380

Corollary 3: Given that the network boundary is sufficiently 381 far away, namely, $R_{\text {nw }} \rightarrow+\infty$, a lower bound for the coverage 382 probability $p_{c}(T, \lambda, \alpha)$ is expressed as

$$
p_{c l}(T, \lambda, \alpha)=\pi \lambda \int_{0}^{R^{2}} e^{-\bar{h} T v^{\alpha / 2} \chi(\alpha, v)-\pi \lambda v} d v
$$

where

$$
\chi(\alpha, v)=P^{-1} \sigma^{2}+\frac{2 \pi \rho \bar{h}}{\alpha-2}\left(2 R-v^{1 / 2}\right)^{2-\alpha} .
$$

Remark 1: In the coverage analysis for the EUDPE-PD 385 model, the average coverage radius $R$ is related to the average 386 cell area $\mathbb{E}\left[A_{s}\right]$. Noting $R \propto \sqrt{\mathbb{E}\left[A_{s}\right]}$ and $\mathbb{E}\left[A_{s}\right]=1 / \rho$, we 387 may use

$$
R=\frac{c_{f}}{\sqrt{\rho}}
$$

where $c_{f}$ is an empirically chosen factor. For example, if the 389 average cell is defined by a square shape, we have $\mathbb{E}\left[A_{s}\right]=390$ $4 R^{2}$; therefore, we have $c_{f}=1 / 2=0.5$. On the other hand, 391 if the average coverage area is calculated according to a hexag- 392 onal one, we have $\mathbb{E}\left[A_{s}\right]=2 \sqrt{3} R^{2}$, yielding $c_{f}=1 / \sqrt{2 \sqrt{3}} \approx 393$ 0.54 , whereas for the average circle-shape cell, we have $c_{f}=394$ $1 / \sqrt{\pi} \approx 0.56$.

\section{B. Coverage Probability Analysis Using EUDPE-UD}

For many practical cellular networks, the geographic BS 397 distribution is often close to a uniform random distribution. 398 Therefore, we next consider the UD BS model with the average 399 
400 density of BSs given by $\rho$. In this case, the corresponding cdf is 401 given by

$$
\mathbb{P}\{z \leq x\}=F(x)=\frac{x^{2}}{c_{\mathrm{nm}}^{2}} \rho, \quad 0 \leq x \leq R
$$

402 where $c_{\mathrm{nm}}^{2}$ is a normalization factor, and $R$ is the coverage 403 radius of the serving BS. Thus, the pdf is given as

$$
f(r)=\frac{2 \rho}{c_{\mathrm{nm}}^{2}} r, \quad 0 \leq r \leq R
$$

404 The normalization factor $c_{\mathrm{nm}}^{2}$ is determined as follows. Assume 405 that $E\left[A_{s}\right]=R^{2} / c_{f}^{2}$, where $c_{f}$ is defined in (26), and fur406 ther note that $E\left[A_{s}\right]=1 / \rho$. From $\int_{0}^{R} f(r) d r=1$, we obtain $407 c_{\mathrm{nm}}^{2}=c_{f}^{2}$.

408 The coverage probability is therefore defined as

$$
\begin{aligned}
p_{c}(T, \rho, \alpha) & =\mathbb{E}_{r}\left[\mathbb{E}_{I_{r}}[\mathbb{P}\{\operatorname{SINR}>T\}]\right] \\
& =\frac{\rho}{c_{f}^{2}} \int_{0}^{R} \mathbb{E}_{I_{r}}\left[\mathbb{P}\left\{h_{0}>P^{-1} \operatorname{Tr}^{\alpha}\left(\sigma^{2}+I_{r}\right)\right\}\right] 2 r d r .
\end{aligned}
$$

409 A lower bound of $\mathbb{E}_{I_{r}}\left[\mathbb{P}\left\{h_{0}>P^{-1} \operatorname{Tr}^{\alpha}\left(\sigma^{2}+I_{r}\right)\right\}\right]$ is given in 410 Theorem 2. Similar to the case of the EUDPE-PD expressed in 411 Theorem 3, therefore, a lower bound for the coverage probabil412 ity $p_{c}(T, \rho, \alpha)$ is given by the following theorem.

413 Theorem 4: For the network where the BS geographic distri414 bution obeys the uniform random distribution with an average 415 BS density of $\rho$, a lower bound for the coverage probability $416 p_{c}(T, \rho, \alpha)$ is given by

$$
p_{c l}(T, \rho, \alpha)=\frac{\rho}{c_{f}^{2}} \int_{0}^{R^{2}} e^{-\bar{h} T v^{\alpha / 2} \psi(\alpha, v)} d v
$$

417 where $\psi(\alpha, v)$ is defined in (22).

418 Corollary 4: Given that the network boundary is sufficiently 419 far away, a lower bound for the coverage probability $p_{c}(T, \rho, \alpha)$ 420 is expressed by

$$
p_{c l}(T, \rho, \alpha)=\frac{\rho}{c_{f}^{2}} \int_{0}^{R^{2}} e^{-\bar{h} T v^{\alpha / 2} \chi(\alpha, v)} d v
$$

421 where $\chi(\alpha, v)$ is defined in (25).

422 Remark 2: How to set the average coverage radius $R$ is 423 explained in Remark 1. Specifically, we may use $R=c_{f} / \sqrt{\rho}$, 424 where $c_{f}$ is an empirically chosen factor.

\section{C. Coverage Probability Analysis Using EUDPE-Grid}

426 With the aid of the EUDPE method, it is straightforward to 427 carry out the coverage probability analysis for all the traditional 428 deterministic grid-based cellular network models, such as the 429 squared and hexagonal ones. This is because the coverage 430 probability analysis using the EUDPE-Grid model is simply a 431 degenerated or special case of the EUDPE-UD-based analysis, where the density of BSs $\rho$ is identical everywhere in the net- 432 work, and every cell has the identical shape with the same area 433 $A_{s}$. Therefore, the lower bounds of the coverage probability for 434 the finite-size and infinite-size grid-based network models are 435 given in Theorem 4 and Corollary 4, respectively. Moreover, 436 choosing $R=1 /(2 \sqrt{\rho})$ corresponds to the grid-based network 437 with squared cells, whereas using $R=1 /(\sqrt{2 \sqrt{3}} \sqrt{\rho})$ is related 438 to considering the grid-based network with hexagonal cells. In 439 general, we may use $R=c_{f} / \sqrt{\rho}$ for any deterministic grid- 440 based network by choosing an appropriate value for $c_{f}$. It be- 441 comes obvious that, under the equivalent network environment 442 of the same $\rho$ and $R$ values, the coverage probability obtained 443 by the EUDPE-Grid-based analysis is identical to that obtained 444 by the EUDPE-UD-based analysis.

\section{Numerical Results for Coverage Probability}

We evaluated the coverage probability first by simulation and 447 used the simulated results as the benchmark for the comparison 448 with our theoretical analytic results. We considered two sce- 449 narios. The first case is a single-tier network constructed by 450 macrocells, obeying the uniform random BS distribution and 451 the cellular channel model described in Section II, whereas 452 the second network followed a Poisson BS distribution and 453 obeyed the same cellular channel model of Section II. Given 454 the SINR threshold $T$, the path-loss exponent $\alpha$, and the SINR 455 value, the simulated coverage probability was calculated using 456 the pseudocodes presented in Algorithm 1. In the simulation, 457 we set the number of BSs to $N_{\mathrm{BS}}=80$, the number of UEs to 458 $N_{\mathrm{UE}}=10000$, the network coverage area to Network Area $=459$ $1000 \times 1000 \mathrm{~m}^{2}$, and the number of sample simulations to 460 $N_{\max }=100$. The average density of BSs was then given as $\quad 461$

$$
\rho=\frac{N_{\mathrm{BS}}}{\text { Network Area }}\left[\mathrm{BSs} / \mathrm{m}^{2}\right] .
$$

For the Poisson distribution, its intensity was $\lambda=\rho$. We com- 462 pared our low-bound coverage probability results based on the 463 EUDPE-PD and EUDPE-UD models with that of the PPP- 464 based analysis [12]. Since the PPP method can only consider 465 the case of an infinitely large network, we assumed the network 466 boundary $R_{\text {nw }} \rightarrow+\infty$. In the following comparison, the simu- 467 lation results obtained by the network with the uniform random 468 BS distribution are labeled as Simulated data 1, whereas the 469 simulation results yielded by the network with the Poisson BS 470 distribution are denoted Simulated data 2.

Algorithm 1 Network Simulation to Evaluate the Coverage Probability.

1: Give the number of BSs $N_{\mathrm{BS}}$, the Network Area, and the 472 number of UEs $N_{\mathrm{UE}}$; $\quad 473$

2: Give the maximum number of sample simulations $N_{\max } ; \quad 474$

3: Set Average Coverage Probability $=0 ; \quad 475$

4: for $N_{\mathrm{sm}}=1$ to $N_{\max }$ do 476

5: Uniformly and randomly draw the $N_{\mathrm{BS}}$ BSs over Net- 477 work Area, or draw the $N_{\mathrm{BS}}$ BSs over Network Area by 478 the Poisson distribution; 


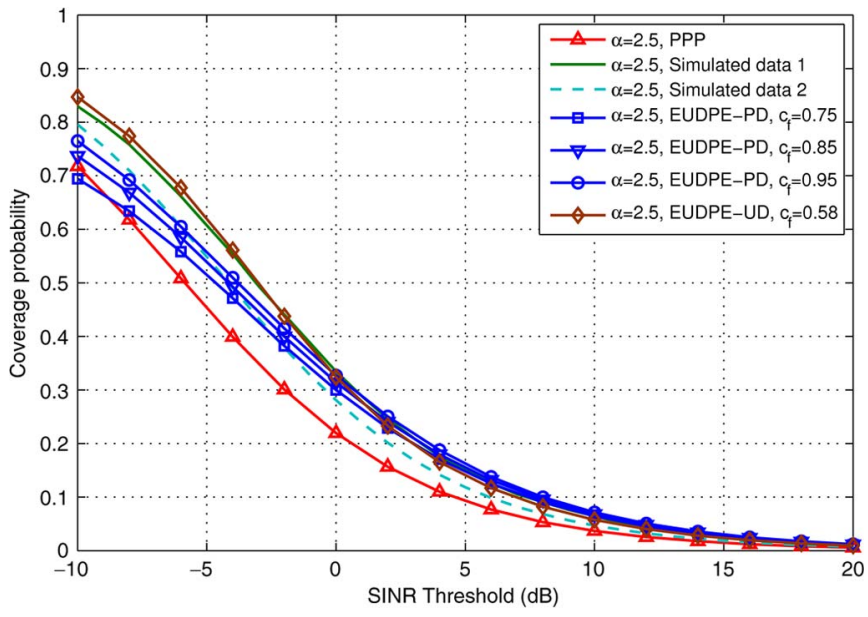

Fig. 7. Comparison of the coverage probabilities based on three different models to the network simulation results, given the path-loss exponent of $\alpha=2.5$ and no noise, i.e., the AWGN power $\sigma^{2}=0$ and SINR $=$ SIR.

480 6: Uniformly and randomly draw the $N_{\text {UE }}$ UEs over Network Area;

482 7: Initialization: count $=0$;

483 8: for $j=1$ to $N_{\mathrm{UE}}$, do

484 9: $\quad$ if $\operatorname{SINR}_{j} \geq T$ then

485 10: $\quad$ count $=$ count +1 ;

end if

486 11: 12 : end for

488 13: Coverage Probability $=$ count $/ N_{\mathrm{UE}}$;

489 14: Average Coverage Probability $+=$

$490 \quad$ Coverage Probability;

491 15: end for

492 16: Average Coverage Probability / $=N_{\max }$.

493 Given the path-loss exponent of $\alpha=2.5$ and assuming no 494 AWGN or $\sigma^{2}=0$, which implies SINR $=$ SIR, Fig. 7 shows 495 the coverage probabilities calculated based on the three analytic 496 models, in comparison to the coverage probabilities obtained by 497 the two different network simulations, when varying the SINR 498 threshold. It is shown in Fig. 7 that the coverage probability 499 analysis results of our proposed EUDPE-PD and EUDPE-UD 500 models agree with both simulation results well, better than the 501 PPP-based analysis. When the path-loss exponent is increased 502 to $\alpha=3$ and 4 , the results obtained are shown in Figs. 8 503 and 9, respectively, where it can be seen that the EUDPE504 UD analysis agrees with the simulation result based on the 505 network with the uniform random BS distribution better than 506 the other two models, whereas the PPP-based analysis agrees 507 better with the simulation result of the network with the Poisson $508 \mathrm{BS}$ distribution better than the other two models.

509 It is worth emphasizing that because there exist no real 510 network performance data to validate an analysis model, we 511 can only rely on the simulated data. When we have an analysis 512 model agrees with a particular simulation result better than an513 other analysis model, it does not imply that the former is better 514 than the latter. The particular simulation result may not actually 515 represent the true real network performance and, moreover, the 516 simulation conditions may not actually match those imposed 517 on an analysis model. What we can claim however is that, if

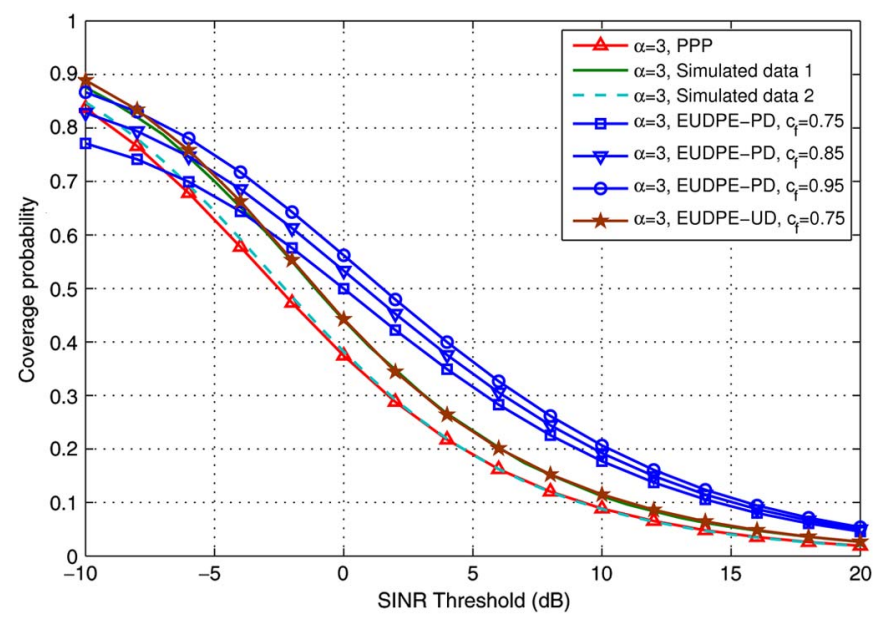

Fig. 8. Comparison of the coverage probabilities based on three different models to the network simulation results, given the path-loss exponent of $\alpha=3$ and no noise, i.e., the AWGN power $\sigma^{2}=0$ and SINR $=$ SIR.

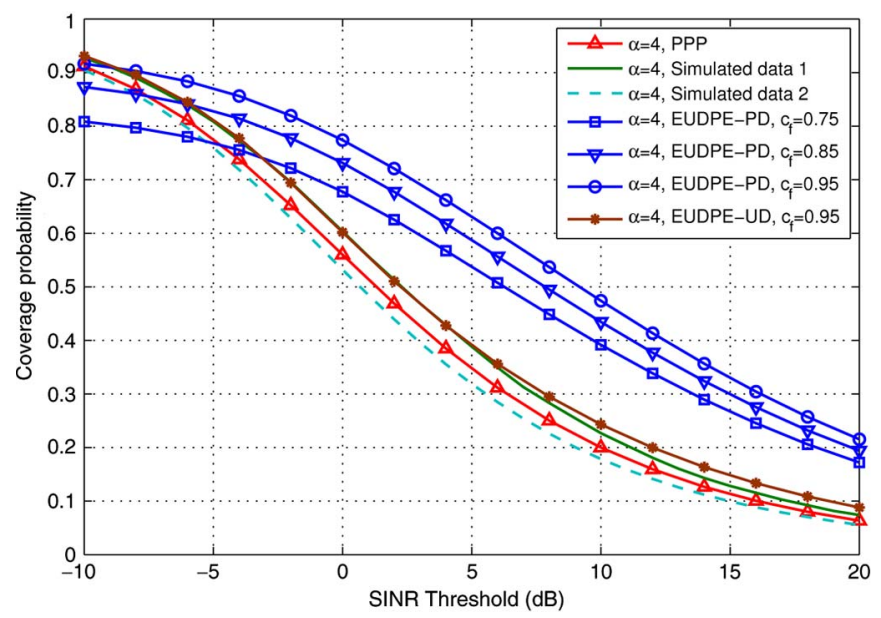

Fig. 9. Comparison of the coverage probabilities based on three different models to the network simulation results, given the path-loss exponent of $\alpha=4$ and no noise, i.e., the AWGN power $\sigma^{2}=0$ and SINR $=$ SIR.

an analysis model agrees well with simulation data, it is a rea- 518 sonable tool for network analysis and planning. Similarly, if a 519 lower bound coverage probability derived by an analysis model 520 appears to be larger than a simulated coverage probability, it 521 does not imply that this analysis model is wrong. Again, the 522 simulation conditions may not actually match those imposed 523 on the analysis model. For example, we assumed that the 524 network boundary $R_{\mathrm{nw}} \rightarrow+\infty$ for the proposed EUDPE-PD 525 and EUDPE-UD models and the PPP-based analysis for the fair 526 comparison of the three analysis models since the PPP method 527 can only be applied for the case of an infinitely large network. 528 However, the simulated network size was $1000 \times 1000 \mathrm{~m}^{2}$ and 529 not infinitely large. As shown earlier, another advantage of 530 our analysis approach over the PPP-based method is that our 531 method can be applied to analyze finite-size networks.

In our EUDPE-based analysis, the empirical chosen factor 533 $c_{f}$ is related to the average cell shape and size. The theoretical 534 explanations of this area factor $c_{f}$ are given in Remark 1.535 Observe from Fig. 7 that, for the path-loss exponent $\alpha=2.5$, an 536 appropriate value of this area factor for our EUDPE-UD model 537 is $c_{f}=0.58$, which is, in fact, close to the case of the average 538 


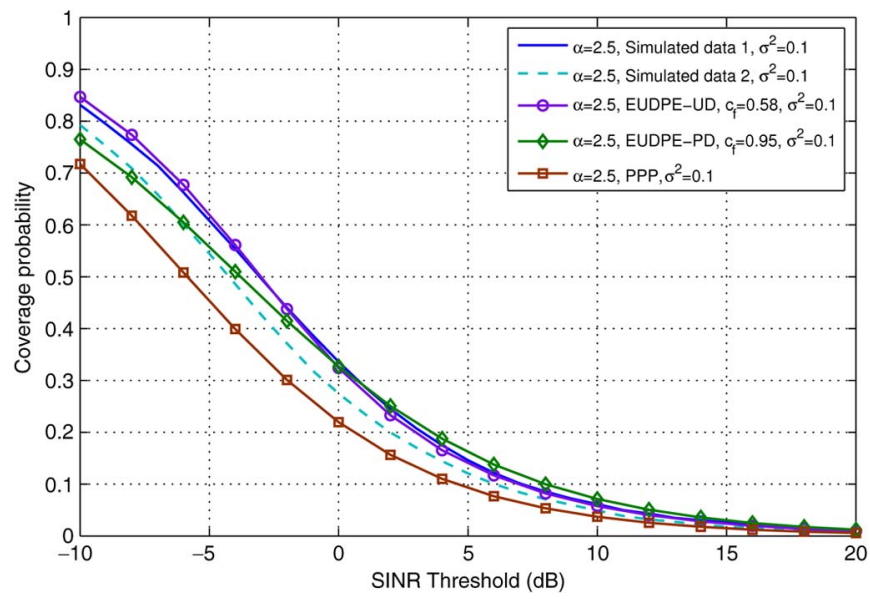

Fig. 10. Comparison of the coverage probabilities based on three different models to the network simulation results, given the path-loss exponent of $\alpha=$ 2.5 and the AWGN power $\sigma^{2}=0.1$.

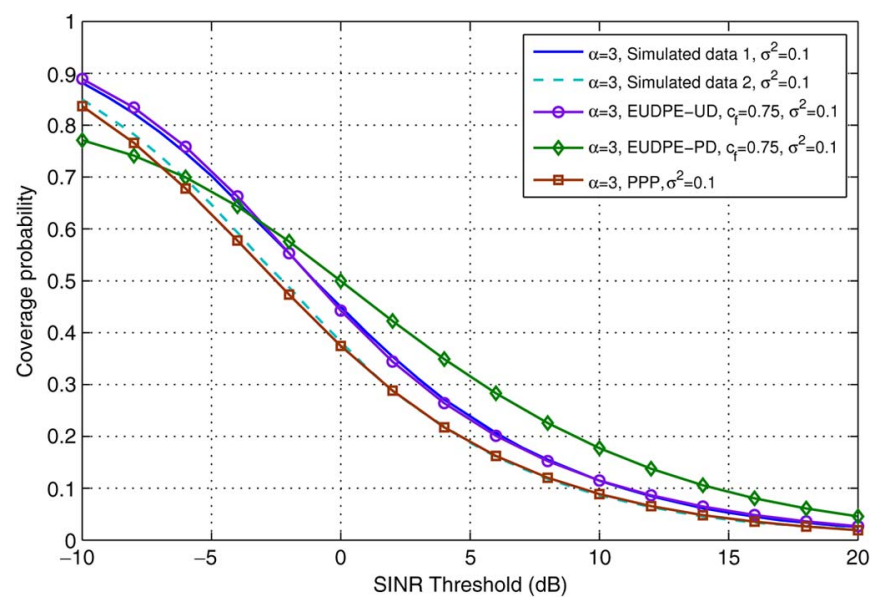

Fig. 11. Comparison of the coverage probabilities based on three different models to the network simulation results, given the path-loss exponent of $\alpha=3$ and the AWGN power $\sigma^{2}=0.1$

539 circle-shaped cell. However, as shown in Figs. 8 and 9, as $\alpha$ 540 increases, the appropriate area factor $c_{f}$ value also increases. A 541 plausible explanation for this phenomenon is offered as follows. 542 As the path-loss exponent $\alpha$ increases, the effective coverage 543 area $R^{2} / c_{f}^{2}$ of the serving BS is reduced, and this corresponds 544 to an increase in the area factor $c_{f}$.

545 Next, the effect of noise imposed on the achievable coverage 546 probability was investigated by setting the AWGN power to $547 \sigma^{2}=0.1$ or $10 \log _{10}\left(1 / \sigma^{2}\right)=10 \mathrm{~dB}$, and the results obtained 548 are given in Figs. 10-12, respectively, for the three differ549 ent values of $\alpha$. For graphic clarity, we only draw a single 550 EUDPE-PD-based coverage probability associated with an ap551 propriate area factor $c_{f}$ value in each of these three figures. 552 Again, the same observations as those drawn for Figs. 7-9 can 553 be made, namely, for the case of $\alpha=2.5$, the EUDPE-UD554 based analysis agrees with the both simulation results better 555 than the PPP-based analysis, whereas for higher $\alpha$ values, the 556 EUDPE-UD analysis matches better with the simulated results 557 based on the uniform random BS distribution, and the PPP558 based analysis agrees better with the simulated results based

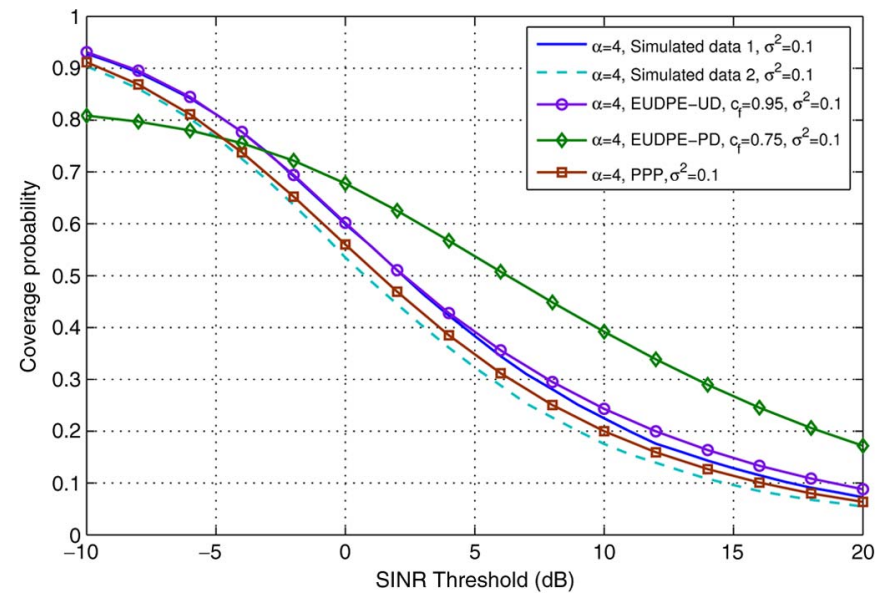

Fig. 12. Comparison of the coverage probabilities based on three different models to the network simulation results, given the path-loss exponent of $\alpha=4$ and the AWGN power $\sigma^{2}=0.1$.

on the Poisson BS distribution. Upon comparing Figs. 10-12 559 with Figs. 7-9, it can be seen that the effect of the channel 560 AWGN to the achievable coverage probability is minor. For 561 example, observe that the simulated-data-2 curve in Fig. 7562 almost matches the simulated-data-2 curve in Fig. 10, whereas 563 the PPP-analysis-based curve in Fig. 7 is almost identical to the 564 PPP-analysis-based curve in Fig. 10. Similarly, the other three 565 coverage probability curves in Fig. 10 also closely match the 566 corresponding coverage probability curves in Fig. 7.

\section{Average Achievable Rate Analysis Using Equivalent Uniform Density Plane-EnTITY}

Let us now apply the proposed EUDPE method to analyze 570 the average achievable throughput. According to Shannon's 571 theory, under the idealized simplifying condition of having a 572 Gaussian interference owing to the central limit theorem, the 573 average achievable rate is defined as [12]

$$
C \triangleq \mathbb{E}[\ln (1+\operatorname{SINR})] .
$$

Since we are concerned with the system's achievable through- 575 put, we will consider the case of the network boundary being 576 sufficiently far away, i.e., $R_{\mathrm{nw}} \rightarrow+\infty$.

\section{A. Average Achievable Rate Analysis Using EUDPE-PD}

Again, we first consider the case that the geographic BS 579 distribution follows a Poisson distribution, and we have the 580 following result.

Theorem 5: For the network where the BS geographic 582 distribution obeys the Poisson distribution of intensity $\lambda$, a 583 lower bound for the average achievable throughput is given by 584

$$
C_{l}(\lambda, \alpha)=\pi \lambda \int_{0}^{R^{2}} e^{-\pi \lambda v}\left(\int_{t>0} e^{-\bar{h} v^{\alpha / 2}\left(e^{t}-1\right) \chi(\alpha, v)} d t\right) d v
$$

where $\chi(\alpha, v)$ is given in (25). 


$$
\begin{aligned}
& C(\lambda, \alpha)=\int_{0}^{R} 2 \pi \lambda r e^{-\pi \lambda r^{2}} \\
& \times \int_{t>0} \mathbb{E}_{I_{r}}\left[\mathbb{P}\left\{h_{0}>P^{-1} r^{\alpha}\left(e^{t}-1\right)\left(\sigma^{2}+I_{r}\right)\right\}\right] d t d r .
\end{aligned}
$$

587 Similar to Corollary 2, we have

$$
\begin{aligned}
\mathbb{E}_{I_{r}}\left[\mathbb { P } \left\{h_{0}>P^{-1} r^{\alpha}\left(e^{t}-1\right)\left(\sigma^{2}\right.\right.\right. & \left.\left.\left.+I_{r}\right)\right\}\right] \\
& \geq e^{-\bar{h} r^{\alpha}\left(e^{t}-1\right) \xi(\alpha, r)}
\end{aligned}
$$

588 where $\xi(\alpha, r)$ is defined in (20). Thus, a lower bound of $C(\lambda, \alpha)$ 589 is given by

$$
C_{l}(\lambda, \alpha)=\int_{0}^{R} 2 \pi \lambda r e^{-\pi \lambda r^{2}}\left(\int_{t>0} e^{-\bar{h} r^{\alpha}\left(e^{t}-1\right) \xi(\alpha, r)} d t\right) d r .
$$

590 By defining $v=r^{2}$ in (37), we obtain (34).

591 Corollary 5: In the noise-free case, namely, $\sigma^{2}=0$, a lower 592 bound for the average achievable throughput is

$$
C_{l}(\lambda, \alpha)=\pi \lambda \int_{0}^{R^{2}} e^{-\pi \lambda v}\left(\int_{t>0} e^{-\bar{h} v^{\alpha / 2}\left(e^{t}-1\right) \bar{\chi}(\alpha, v)} d t\right) d v
$$

593 where

$$
\bar{\chi}(\alpha, v)=\frac{2 \pi \rho \bar{h}}{\alpha-2}\left(2 R-v^{1 / 2}\right)^{2-\alpha} .
$$

\section{B. Average Achievable Rate Analysis Using EUDPE-UD}

595 Next, we consider the case that the geographic BS distribu596 tion follows a uniform random distribution, and we have the 597 following result.

598 Theorem 6: For the network where the BS geographic dis599 tribution obeys the uniform random distribution with an average 600 BS density of $\rho$, a lower bound for the average achievable 601 throughput is given by

$$
C_{l}(\rho, \alpha)=\frac{\rho}{c_{f}^{2}} \int_{0}^{R^{2}}\left(\int_{t>0} e^{-\bar{h} v^{\alpha / 2}\left(e^{t}-1\right) \chi(\alpha, v)} d t\right) d v
$$

602 where $\chi(\alpha, v)$ is given in (25).

603 Proof: Noting that the average achievable throughput is 604 defined as

$$
\begin{aligned}
& C(\lambda, \alpha)=\frac{\rho}{c_{f}^{2}} \int_{0}^{R} 2 r \\
& \times \int_{t>0} \mathbb{E}_{I_{r}}\left[\mathbb{P}\left\{h_{0}>P^{-1} r^{\alpha}\left(e^{t}-1\right)\left(\sigma^{2}+I_{r}\right)\right\}\right] d t d r
\end{aligned}
$$

605 the proofs are similar to the proofs for Theorem 5.
Corollary 6: In the noise-free case, namely, $\sigma^{2}=0$, a lower 606 bound for the average achievable throughput is

$$
C_{l}(\rho, \alpha)=\frac{\rho}{c_{f}^{2}} \int_{0}^{R^{2}}\left(\int_{t>0} e^{-\bar{h} v^{\alpha / 2}\left(e^{t}-1\right) \bar{\chi}(\alpha, v)} d t\right) d v .
$$

where $\bar{\chi}(\alpha, v)$ is given in (39).

608

Remark 3: It is straightforward to carry out the average 609 achievable throughput analysis for any deterministic grid-based 610 cellular network model, because the EUDPE-Grid model is a 611 special case of the EUDPE-UD model. Therefore, the lower 612 bound of the average achievable throughput for the grid-based 613 network model is also given in Theorem 6. Moreover, under the 614 equivalent network environment of the same $\rho$ and $R$ values, 615 the lower bound of the average achievable throughput obtained 616 by the EUDPE-Grid-based analysis is identical to that obtained 617 by the EUDPE-UD-based analysis.

\section{Numerical Results for Average Achievable Rate}

Assuming a unity frequency reuse factor, we compare the 620 lower bounds of the average achievable throughput obtained 621 by the proposed EUDPE-PD- and EUDPE-UD-based analyses 622 to that of the PPP-based analysis [12] in Fig. 13 by varying 623 the path-loss exponent value. The simulated average achiev- 624 able throughputs obtained from the two network simulations 625 with the uniform random BS distribution and the Poisson BS 626 distribution are labeled as Simulated rate 1 and Simulated 627 rate 2, respectively, and they are also given in Fig. 13 as the 628 benchmark. For our proposed EUDPE-PD and EUDPE-UD- 629 based analysis and the network simulations, both the noise- 630 free and noisy results are presented. However, for the 631 PPP-based average achievable throughput analysis, only the 632 noise-free case is provided in [12]; therefore, in Fig. 13, we only 633 present the noise-free PPP-based result. It can be observed that 634 all the three theoretical analysis based results and the simulation 635 data all reveal that the average achievable throughput increases 636 linearly, as the path-loss exponent increases. More specifically, 637 all the analytical and simulated data have accurate linear fitting. 638 It is also shown in Fig. 13 that our proposed EUDPE-PD- 639 and EUDPE-UD-based analyses agree with the two simulated 640 results better than the PPP-based analysis, particularly for the 641 path-loss exponent $\alpha \leq 4.5$. The results of Fig. 13 also show 642 that the noise only has a minor effect on the average achievable 643 throughput, which is expected as we consider the interference- 644 limited scenario with a unity frequency reuse factor.

\section{CONCLUSION}

646

We have proposed a universal approach for accurately 647 analyzing the coverage probability and average achievable 648 throughput of cellular networks. More specifically, we have 649 derived a generic EUDPE procedure for evaluating the other- 650 cell interference. Based on this EUDPE interference model, we 651 have derived the lower bounds of both the coverage probability 652 and average achievable throughput for various practical BS 653 distribution models, including the stochastic Poisson distributed 654 model, uniformly and randomly distributed model, and the 655 


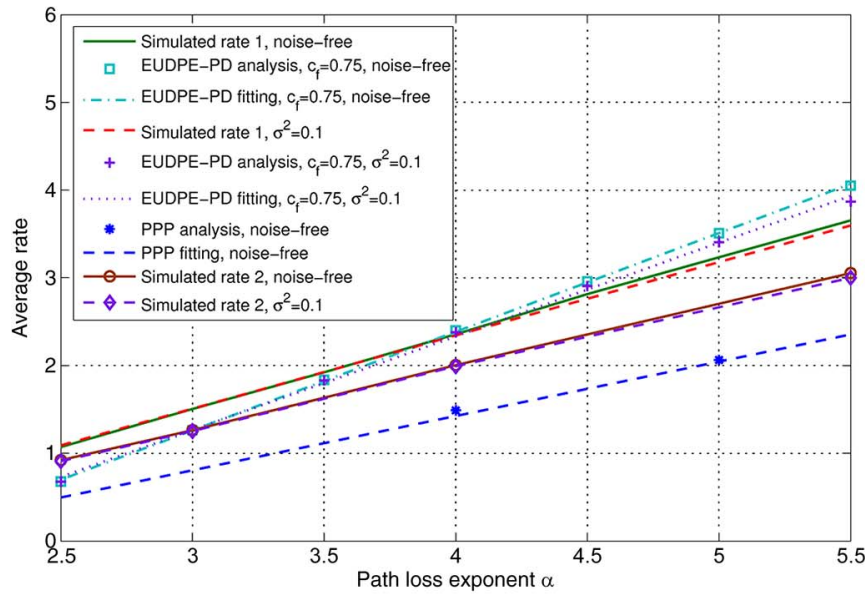

(a)

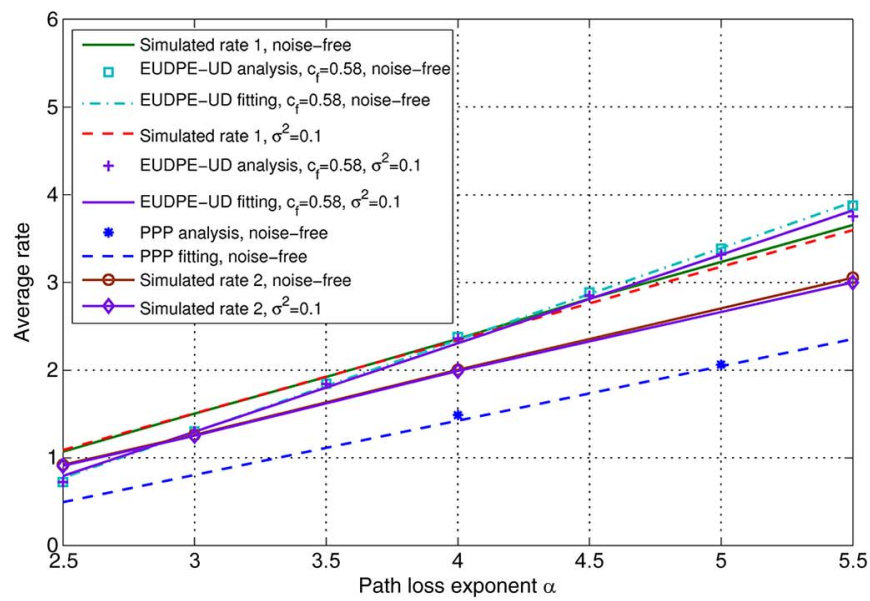

(b)

Fig. 13. Comparison of the average achievable throughputs based on three different models to the network simulation results, given different path-loss exponent values. (a) EUDPE-PD and PPP models and (b) EUDPE-UD and PPP models.

656 deterministic grid-based model. Extensive simulation results 657 have validated that the coverage probability and average 658 throughput obtained by our proposed universal analysis method 659 agree with the simulated coverage probability and average 660 throughput at least as closely as those obtained by the popular 661 existing PPP-based analysis, if not better. In addition, we have 662 also introduced a generic and physical definition of cell edge 663 boundary. We have shown that the popular hexagonal and 664 Voronoi network topologies only emerge from the grid-based 665 network model and the random BS distribution model, respec666 tively, given an unrealistic high path-loss exponent according 667 to this definition. Moreover, we have demonstrated that the cell 668 edge boundary shows irregular near-circular shapes, given a 669 more realistic path-loss exponent, which cannot be modeled 670 accurately by either hexagonal or Voronoi topology.

\section{REFERENCES}

[1] A. Damnjanovic et al., "A survey on 3GPP heterogeneous networks," IEEE Wireless Commun., vol. 18, no. 3, pp. 10-21, Jun. 2011.

[2] J. G. Andrews, "Seven ways that hetnets are a cellular paradigm shift," IEEE Commun. Mag., vol. 51, no. 3, pp. 136-144, Mar. 2013.

[3] A. Ghosh, et al., "Heterogeneous cellular networks: From theory to practice," IEEE Commun. Mag., vol. 50, no. 6, pp. 54-64, Jun. 2012.
[4] J. G. Andrews, H. Claussen, M. Dohler, S. Rangan, and M. C. Reed, 678 "Femtocells: Past, present, and future," IEEE J. Sel. Areas Commun., 679 vol. 30, no. 3, pp. 497-508, Apr. 2012.

[5] X. Yang and A. P. Petropulu, "Co-channel interference modeling and 681 analysis in a Poisson field of interferers in wireless communications," 682 IEEE Trans. Signal Process., vol. 51, no. 1, pp. 64-76, Jan. 2003.

[6] A. D. Wyner, "Shannon-theoretic approach to a Gaussian cellular 684 multiple-access channel," IEEE Trans. Inf. Theory, vol. 40, no. 6, 685 pp. 1713-1727, Nov. 1994

686

[7] S. Shamai and A. D. Wyner, "Information-theoretic considerations for 687 symmetric, cellular, multiple-access fading channels, Part I," IEEE Trans. 688 Inf. Theory, vol. 43, no. 6, pp. 1877-1894, Nov. 1997.

[8] S. Shamai and A. D. Wyner, "Information-theoretic considerations 690 for symmetric, cellular, multiple-access fading channels, Part II," IEEE 691 Trans. Inf. Theory, vol. 43, no. 6, pp. 1895-1911, Nov. 1997.

[9] J. Xu, J. Zhang, and J. G. Andrews, "On the accuracy of the Wyner 693 model in cellular networks," IEEE Trans. Wireless Commun., vol. 10, 694 no. 9, pp. 3098-3109, Sep. 2011.

695

[10] F. Baccelli, M. Klein, M. Lebourges, and S. Zuyev, "Stochastic 696 geometry and architecture of communication networks," Telecommun. 697 Syst., vol. 7, no. 1-3, pp. 209-227, Jun. 1997.

698

[11] T. X. Brown, "Cellular performance bounds via shotgun cellular 699 systems," IEEE J. Sel. Areas Commun., vol. 18, no. 11, pp. 2443-2455, 700 Nov. 2000.

[12] J. G. Andrews, F. Baccelli, and R. K. Ganti, "A tractable approach to 702 coverage and rate in cellular networks," IEEE Trans. Commun., vol. 59, 703 no. 11 , pp. 3122-3134, Nov. 2011.

704

[13] R. W. Heath and M. Kountouris, "Modeling heterogeneous network 705 interference," in Proc. IEEE ITA, San Diego, CA, USA, Feb. 5-10, 2012, 706 pp. 17-22.

[14] H. S. Dhillon, R. K. Ganti, F. Baccelli, and J. G. Andrews, "Modeling 708 and analysis of K-tier downlink heterogeneous cellular networks," IEEE 709 J. Sel. Areas Commun., vol. 30, no. 3, pp. 550-560, Apr. 2012.

[15] M. Z. Win, P. C. Pinto, and L. A. Shepp, "A mathematical theory 711 of network interference and its applications," Proc. IEEE, vol. 97, no. 2, 712 pp. 205-230, Feb. 2009.

713

[16] A. Busson and G. Chelius, "Point processes for interference modeling 714 in CSMA/CA ad-hoc networks," in Proc. 6th ACM Symp. Performance 715 Eval. Wireless Ad Hoc, Sens., Ubiquitous Netw., Tenerife, Canary Islands, 716 Spain, Oct. 26-30, 2009, pp. 33-40.

717

[17] F. Baccelli, B. Błaszczyszyn, and P. Mühlethaler, "An Aloha protocol 718 for multihop mobile wireless networks," IEEE Trans. Inf. Theory, vol. 52, 719 no. 2, pp. 421-436, Feb. 2006

720

[18] H. Q. Nguyen, F. Baccelli, and D. Kofman, "A stochastic geometry 721 analysis of dense IEEE 802.11 networks," in Proc. 26th IEEE INFOCOM, 722 Anchorage, AK, USA, May 6-12, 2007, pp. 1199-1207.

[19] M. L. Huber and R. L. Wolpert, "Likelihood-based inference for Matérn 724 type-III repulsive point processes," Adv. Appl. Probab., vol. 41, no. 4, 725 pp. 958-977, Dec. 2009.

726

[20] M. Haenggi, "Mean interference in hard-core wireless networks," IEEE 727 Commun. Lett., vol. 15, no. 8, pp. 792-794, Aug. 2011.

[21] A. Hasan and J. G. Andrews, "The guard zone in wireless ad hoc 729 networks," IEEE Trans. Wireless Commun., vol. 6, no. 3, pp. 897-906, 730 Mar. 2007.

731

[22] B. Cho, K. Koufos, and R. Jantti, "Bounding the mean interference 732 in Matérn type II hard-core wireless networks," IEEE Wireless Commun. 733 Lett., vol. 2, no. 5, pp. 563-566, Oct. 2013.

[23] F. Jarai-Szabo and Z. Neda, "On the size-distribution of Poisson 735 Voronoi cells," Phys. A, Statist. Mech. Appl., vol. 385, no. 2, pp. 518-526, 736 Feb. 2007.

[24] D. Cao, S. Zhou, and Z. Niu, "Optimal combination of base sta- 738 tion densities for energy-efficient two-tier heterogeneous cellular net- 739 works," IEEE Trans. Wireless Commun., vol. 12, no. 9, pp. 4350-4362, 740 Sep. 2013.

[25] S. Lee and K. Huang, "Coverage and economy of cellular networks with 742 many base stations," IEEE Commun. Lett., vol. 16, no. 7, pp. 1038-1040, 743 Jul. 2012.

744

26] J.-M. Kelif, M. Coupechoux, and P. Godlewski, "A fluid model for per- 745 formance analysis in cellular networks," EURASIP J. Wireless Commun. 746 Netw., vol. 2010, pp. 1-11, Aug. 2010.

[27] F. Baccelli and B. Blaszczyszyn, Stochastic Geometry and Wireless 748 Networks, Volume I: Theory. Hanover, MA, USA: Now, 2009.

[28] M. Haenggi, Stochastic Geometry for Wireless Networks, Cambridge, 750 U.K.: Cambridge Univ. Press, 2013.

[29] M. Kuczma, An Introduction to the Theory of Functional Equations 752 and Inequalities: Cauchy's Equation and Jensen's Inequality. Basel, 753 Switzerland: Birkhauser, 2008. 


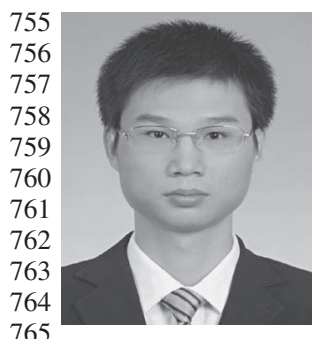

Hui Zhang received the B.Eng. degree in applied mathematics and the Ph.D. degree in electrical engineering from Beijing University of Posts and Telecommunications, Beijing, China, in 2005 and 2010, respectively.

Since 2010, he has been a faculty member with the School of Electrical Information and Optical Engineering, Nankai University, Nankai, China. From 2013 to 2014, he was a Postdoctoral Scholar with the School of Electrical and Computer Science, University of Southampton, Southampton, U.K. In 2014, he 766 joined in the China-Korea Young Scientist Exchange Program. His research 767 interests include cellular networks and wireless communication theory.

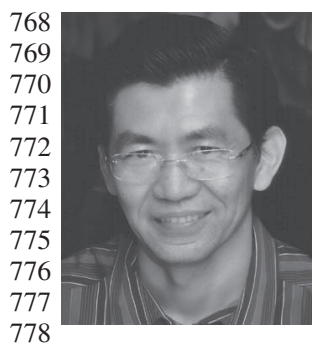

Sheng Chen (M'90-SM'97-F'08) received the B.Eng. degree in control engineering from the East China Petroleum Institute, Dongying, China, in 1982; the Ph.D. degree in control engineering from the City University London, London, U.K., in 1986; and the D.Sc. degree from the University of Southampton, Southampton, U.K., in 2005.

From 1986 to 1999, he held research and academic appointments with The University of Sheffield, Sheffield, U.K.; The University of Edinburgh, Edinburgh, U.K.; and the University of 779 Portsmouth, Portsmouth, U.K. Since 1999, he has been with Electronics 780 and Computer Science, the University of Southampton, Southampton, 781 U.K., where he is currently a Professor of intelligent systems and signal 782 processing. He is also a Distinguished Adjunct Professor with King Abdulaziz 783 University, Jeddah, Saudi Arabia. He is the author of over 500 research 784 papers. His research interests include adaptive signal processing, wireless 785 communications, modeling and identification of nonlinear systems, neural 786 network and machine learning, intelligent control system design, and 787 evolutionary computation methods and optimization.

788 Dr. Chen is a Fellow of the Institution of Engineering and Technology and 789 an ISI highly cited researcher in engineering (March 2004). In 2014, he was 790 elected as a Fellow of the United Kingdom Royal Academy of Engineering.

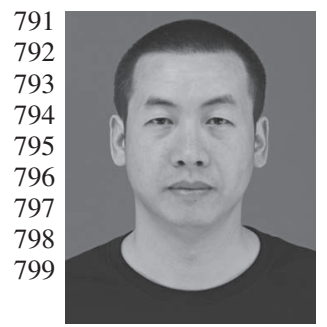

Liang Feng received the B.Eng. degree in applied physics from Dalian University of Technology, Dalian, China, in 2004. He is currently working toward the Master's degree with Nankai University, Nankai, China, in 2012.

From 2004 to 2012, he carried out electronic countermeasures research with the Luoyang Electronic Equipment Center, China. His research interests include cellular mobile communications.

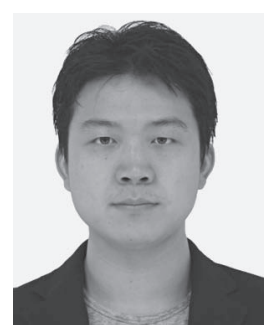

Yifeng Xie received the B.S. degree in electronic 800 and information engineering from Nanjing Forestry 801 University, Nanjing, China, in 2011 and the M.S. 802 degree in communication and information systems 803 from Nankai University, Nankai, China, in 2014. 804

His research interests include stochastic geometry 805 and heterogeneous networks. 806

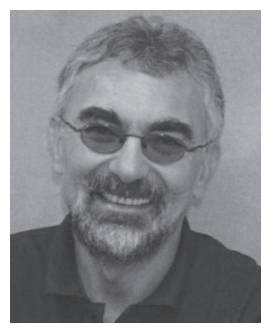

Lajos Hanzo (F'08) received the Master's and 807 D.Sc. degrees in electronics and the Doctor Honoris 808 Causa from the Technical University of Budapest, 809 Budapest, Hungary, in 1976 and 1983, respectively. 810

During his 38-year career in telecommunications, 811 he has held various research and academic posts in 812 Hungary, Germany, and the U.K. Since 1986, he has 813 been with the School of Electronics and Computer 814 Science, University of Southampton, Southampton, 815 U.K., where he is the Chair in telecommunications. 816 $\mathrm{He}$ has successfully supervised about 100 Ph.D. 817 students. He is the author or coauthor of 20 John Wiley/IEEE Press books 818 on mobile radio communications, totalling in excess of 10000 pages, and of 819 more than 1400 research entries on IEEE Xplore. Currently, he is directing a 820 100 -strong academic research team, working on a range of research projects 821 in the field of wireless multimedia communications sponsored by industry, 822 the Engineering and Physical Sciences Research Council (EPSRC) U.K., the 823 European Research Council's Advanced Fellow Grant, and the Royal Society's 824 Wolfson Research Merit Award. He is an enthusiastic supporter of industrial 825 and academic liaison, and he offers a range of industrial courses.

Dr. Hanzo has acted both as a Technical Program Committee Chair and as a 827 General Chair of IEEE conferences, has presented keynote lectures, and has 828 received a number of distinctions. He is a Governor of the IEEE Vehicular 829 Technology Society. From 2008 to 2012, he was the Editor-in-Chief of the IEEE 830 Press and a Chaired Professor with Tsinghua University, Beijing, China. His 831 research is funded by the European Research Council's Senior Research Fellow 832 Grant. He is a Fellow of the Royal Academy of Engineering, the Institution of 833 Engineering and Technology, and the European Association for Research and 834 Signal Processing. 


\section{AUTHOR QUERIES}

\section{AUTHOR PLEASE ANSWER ALL QUERIES}

AQ1 = Note that Ref. [7] was split into two. Consequently, the reference list and bibliographic citations were renumbered. Please check.

END OF ALL QUERIES 


\title{
- A Universal Approach to Coverage Probability and Throughput Analysis for Cellular Networks
}

\author{
Hui Zhang, Sheng Chen, Fellow, IEEE, Liang Feng, Yifeng Xie, and Lajos Hanzo, Fellow, IEEE
}

\begin{abstract}
4 Abstract-This paper proposes a novel tractable approach 5 for accurately analyzing both the coverage probability and the 6 achievable throughput of cellular networks. Specifically, we de7 rive a new procedure referred to as the equivalent uniform8 density plane-entity (EUDPE) method for evaluating the other-cell 9 interference. Furthermore, we demonstrate that our EUDPE 10 method provides a universal and effective means to carry out the 11 lower bound analysis of both the coverage probability and the 12 average throughput for various base-station distribution models 13 that can be found in practice, including the stochastic Poisson 14 point process (PPP) model, a uniformly and randomly distributed 15 model, and a deterministic grid-based model. The lower bounds 16 of coverage probability and average throughput calculated by our 17 proposed method agree with the simulated coverage probability 18 and average throughput results and those obtained by the existing 19 PPP-based analysis, if not better. Moreover, based on our new 20 definition of cell edge boundary, we show that the cellular topology 21 with randomly distributed base stations (BSs) only tends toward 22 the Voronoi tessellation when the path-loss exponent is suffi23 ciently high, which reveals the limitation of this popular network 24 topology.
\end{abstract}

25 Index Terms-Achievable throughput, cellular coverage, cellu26 lar networks, deterministic grid-based model, Poisson point pro27 cess (PPP) model, uniformly and randomly distributed model.

\section{INTRODUCTION}

30 INCE cellular systems are under growing pressure to in$31-$ crease the volume of data delivered to consumers, es32 tablishing an accurate performance prediction model is of 33 prime significance [1]. Cellular systems are evolving into a 34 large-scale heterogeneous network architecture, constructed by 35 overlapping network tiers, such as macrocells, picocells, fem36 tocells, etc. [2]-[4]. The traditional cellular analysis relying on 37 an idealized hexagonal model does not realistically represent 38 the actual distribution of cells. Clearly, such a simplistic model

Manuscript received March 5, 2014; revised August 31, 2014; accepted October 28, 2014. This work was supported in part by the National Natural Science Foundation Project of China under Grant 61101084 and in part by the Fundamental Research Funds for the Central Universities, China. The review of this paper was coordinated by Dr. Y. Ma.

H. Zhang, L. Feng, and Y. Xie are with the Wireless Communications Laboratory, Information College, Nankai University, Tianjin 300071, China (e-mail: zhangh@nankai.edu.cn; fengliang201203@gmail.com; xyfhope@gmail.com).

S. Chen is with the School of Electronics and Computer Science, University of Southampton, Southampton SO17 1BJ, U.K., and also with King Abdulaziz University, Jeddah 21589, Saudi Arabia (e-mail: sqc@ecs.soton.ac.uk).

L. Hanzo is with School of Electronics and Computer Science, University of Southampton, Southampton SO17 1BJ, U.K. (e-mail: 1h@ecs.soton.ac.uk).

Color versions of one or more of the figures in this paper are available online at http://ieeexplore.ieee.org.

Digital Object Identifier 10.1109/TVT.2014.2366597 cannot be used for accurately modeling real-world cellular 39 networks and for analyzing the coverage probability and the 40 achievable throughput. Two mathematical models, i.e., the cel- 41 lular system interference model and the base station (BS) or cell 42 distribution model, are fundamental in the coverage analysis. 43

\section{A. Related Work and Motivation}

According to [5], the interference models can generally be di- 45 vided into two types: empirical models and statistical-physical 46 models. The construction of an empirical interference model 47 relies on first measuring the interference and then fitting a 48 mathematical model to the data. By contrast, the derivation of 49 a statistical-physical model usually relies on the mathematical 50 modeling of the interference. The classic Wyner model [6] was 51 proposed in 1994, and since then, it has been widely adopted in 52 the analysis of cellular networks. This model assumes that the 53 interference is constituted by the sum of the signals transmitted 54 from the adjacent cells (typically only considering two neigh- 55 bors), which is often multiplied by a fixed scaling factor or gain 56 to represent the specific intensity of the interferers [6]-[9]. $\quad 57$

Determining the most beneficial positions of the BSs rep- 58 resents a critical planning problem in cellular networks. 59 Traditional methods usually place the BSs deterministically on 60 a regular grid, despite the fact that, in practice, the positions 61 of BSs are influenced by many random factors. Taking into 62 account the randomness of BS locations, in [10] and [11], a 63 stochastic-geometry-based method for modeling the positions 64 of the BSs was derived, whereas in [12] and [13], it was 65 proposed that the BSs be placed according to a homogeneous 66 Poisson point process (PPP) associated with a given intensity 67 [12], [13]. However, since the cellular network is gradually 68 evolving into a large-scale heterogeneous network associated 69 with multiple-tier random BS locations, the design challenge 70 becomes more grave. A recent contribution [14] has demon- 71 strated that the BS locations may be drawn from a PPP, partic- 72 ularly for single-tier networks. In [5], the statistical-physical 73 modeling of cochannel interference (CCI) was investigated 74 by assuming that the geographic distribution of interferers is 75 known a priori and that the interferers belong to a Poisson 76 field, with each individual interferer having a random session 77 life time. In [15], a mathematical theory based on a spatially 78 homogeneous PPP was provided to analyze the effects of 79 interference, which models the spatial distribution of the nodes 80 over the 2-D infinite plane by PPP theory.

The PPP model was used in [12] for establishing a hetero- 82 geneous network model of a single-tier macrocell network. 83 
84 Based on this PPP model, the calculation of the cumulative 85 interference imposed by all surrounding BSs can be carried 86 out with the aid of the Laplace transform and the probability 87 generating function [12], [14]. Furthermore, the coverage prob88 ability expression was deduced for the specific scenario, when 89 the interference experiences Rayleigh fading, and the results 90 of [12] and [14] demonstrated that the analysis based on the 91 PPP-aided modeling represent the lower bound of simulation 92 results. ${ }^{1}$ Similarly, the achievable average rate was also calcu93 lated. Although the PPP model is adopted for the analysis of 94 cellular networks, it is only accurate for sparse networks. By 95 contrast, it suffers from a lack of realism in the case of dense 96 networks since it may place several BSs far too closely together, 97 which does not make practical sense as such a situation will not 98 occur in a real BS deployment. It may impose excessive CCI if 99 too many BSs are deployed too densely. Noting this weakness 100 of the PPP model, some balanced measures are suggested to 101 alleviate this drawback in [12], but this weakness cannot be fun102 damentally eliminated by these measures. Moreover, the PPP103 based analysis relies on the assumption that the transmitters are 104 independently distributed [16].

105 A range of alternative stochastic-geometry-based methods 106 have also been used in the analysis of wireless networks [17], 107 [18]. For example, in [17], the Matérn hard-core process was 108 invoked for modeling the classic carrier sense multiple access 109 (CSMA) protocol and for analyzing its throughput, where the 110 presence of interferers within a given radius around any trans111 mitter was prevented. The Matérn point process [19] was modi112 fied in [18] to model the CSMA with collision avoidance, which 113 yields more realistic results by applying the aforementioned 114 interference-exclusion zone around all possible transmitters. 115 However, coverage analysis based on a Matérn hard-core pro116 cess is difficult to carry out [20] since the probability generating 117 functional of a Matérn hard-core process does not exist. It was 118 argued in [20]-[22] that only the Matérn type II process causes 119 a level of interference comparable to that predicted by a PPP 120 and, therefore, for interference-based performance analysis, the 121 Matérn type II process may be safely approximated by the 122 corresponding nonhomogeneous PPP [20]-[22].

\section{B. Our Approach and Contributions}

124 Against the above background, we propose a novel universal 125 approach for tractable and accurate coverage analysis of cellu126 lar networks. Our contributions are as follows.

127 1) Physical Analysis of Hexagonal/Voronoi Cells: To inter128 pret the various geometric-based cellular models from a physi129 cal perspective, we provide a tangible generic definition of the 130 cell edge boundary for our theoretical analysis, where the cell 131 boundary is directly linked to the path-loss exponent. Specif132 ically, we show that the traditional hexagonal topology natu133 rally emerges from the grid-based model, given a sufficiently 134 high path-loss exponent, whereas the Voronoi tessellation nat135 urally emerges from the random BS distribution model, again

\footnotetext{
${ }^{1}$ The simulation results are referred to as "experimental" or "actual" in [12], which is inappropriate.
}

provided that the path-loss exponent is sufficiently high. How- 136 ever, such a high path-loss exponent is unrealistic in real trans- 137 mission environments. Therefore, our physical analysis reveals 138 the fundamental limitation of these purely graphic-based cellu- 139 lar topologies, namely, lack of the connection to the underlying 140 signal transmission medium. In fact, we demonstrate that the 141 cell edge boundary shows irregular near-circular shapes, given a 142 more realistic path-loss exponent of around 3, which cannot be 143 modeled accurately by either hexagonal or Voronoi tessellation. 144

2) EUDPE-Based Other-Cell Interference Model: We pro- 145 pose a universal model for evaluating the other-cell interfer- 146 ence, which we refer to as the equivalent uniform-density 147 plane-entity (EUDPE) method. This generic EUDPE model can 148 be used to calculate the cumulative other-cell interference for 149 all the existing BS distribution models that can be found in 150 practice, including both stochastic and deterministic cellular 151 network models, such as the stochastic Poisson distributed (PD) 152 and uniformly distributed (UD) BS models and the determinis- 153 tic grid-based BS model.

3) Lower Bound Analysis for Coverage Probability and 155 Average Achievable Rate: Based on the proposed generic 156 EUDPE interference model, we perform the low-bound anal- 157 ysis of both the coverage probability and the average achiev- 158 able rate for various BS distribution models, specifically, the 159 stochastic PD and UD BS models and the deterministic grid- 160 based BS model, which may be viewed as a degenerated or spe- 161 cial case of the UD BS model. For realistic path-loss exponents, 162 the coverage probability and average achievable throughput 163 results provided by our proposed analysis approach agree with 164 the simulated coverage probability and achievable throughput. 165 In fact, their match is as good or better than that of the PPP- 166 based analysis. The results also show that the noise only has a 167 modest effect on the coverage probability and achievable rate. 168

The remainder of this paper is organized as follows. In 169 Section II, the downlink cellular system model is briefly in- 170 troduced, which is followed by our new physical analysis of 171 cell edge boundary. Section III is devoted to the derivation of 172 our EUDPE-based interference model. The low-bound analysis 173 of the coverage probability based on the EUDPE method is 174 deduced in Section IV for both stochastic BS distribution 175 models and deterministic grid-based BS models, whereas the 176 corresponding low-bound analysis is presented in Section V. 177 Our conclusions are offered in Section VI.

\section{Downlink Cellular System Model}

Throughout our discussions, the index set of the BSs, which 180 are deployed according to some distribution, is denoted by $\Phi, 181$ whereas the serving BS's index is denoted by $b_{0}$. Furthermore, 182 the average density of BSs is $\rho$. Let $P$ be the transmitted power 183 of a BS, $R$ be the serving BS's coverage radius, $R_{\text {nw }}$ be the 184 distance from the serving BS to the edge of the network, and 185 $r_{i}$ denotes the distance from the $i$ th $\mathrm{BS}$ to the user equipment 186 (UE) concerned. If we denote the average coverage area of a 187 BS by $\mathbb{E}\left[A_{s}\right]$ with $\mathbb{E}[]$ representing the expectation operator, 188 then $\mathbb{E}\left[A_{s}\right]=1 / \rho$. We will also use $2 R$ to denote the average 189 distance between two neighboring BSs, and we have $R \propto 190$ $\sqrt{\mathbb{E}\left[A_{s}\right]}$. 


\section{A. SINR Model}

193 The wireless channel linking the $i$ th BS and the UE con194 cerned is modeled by a complex-valued channel tap that takes 195 into account the path loss with a path-loss exponent of $\alpha$, the 196 fast Rayleigh fading coefficient with an instantaneous power 197 or a squared magnitude of $h_{i}$, and the channel's additive white 198 Gaussian noise (AWGN) with noise power of $\sigma^{2}$. The average 199 of the random variable $h_{i}$ is denoted by $\bar{h}$; therefore, $h_{i}$ follows 200 the exponential distribution with the mean $\bar{h}$.

201 Let us assume that the intracell UE-to-UE interference is neg202 ligible. Then, the signal-to-interference-plus-noise ratio (SINR) 203 experienced at this UE can be expressed as follows:

$$
\mathrm{SINR}=\frac{P h_{0} r_{0}^{-\alpha}}{I_{r}+\sigma^{2}}
$$

204 where the interference arriving from all the interfering cells is 205 given by

$$
I_{r}=\sum_{i \in \Phi \backslash b_{0}} P h_{i} r_{i}^{-\alpha}
$$

206 If the target SINR value is $T$, then the actual SINR must obey 207 SINR $>T$, which requires

$$
h_{0}>P^{-1} \operatorname{Tr}_{0}^{\alpha}\left(\sigma^{2}+I_{r}\right) .
$$

208 Thus, the probability distribution of $h_{0}$ should be taken into 209 account in the analysis of both the coverage probability and the 210 average rate. Furthermore, intuitively, the given SINR model 211 determines the coverage area of each BS; therefore, it influences 212 the cell shape or boundary.

\section{B. Physical Analysis of Cell Edge Boundary}

214 As aforementioned, the grid-based cellular model is conve215 nient but is too idealistic. By contrast, the Voronoi tessellation is 216 considered to match the random BS deployment in relative flat 217 urban areas reasonably well [12], [23]. Hence, cellular networks 218 can be analyzed using Voronoi diagram theory, albeit this has 219 not been explained with the aid of a physically tangible per220 spective. More specifically, both the energy efficiency and cov221 erage of cellular networks may be analyzed based on Voronoi 222 tessellation [24], [25]. Fig. 1 shows a random distribution of 223 the BSs with the cell boundaries corresponding to a Voronoi 224 tessellation. Note that in both the grid-based and Voronoi-based 225 cellular topologies, the cell boundaries are determined purely 226 by the geometric property of the BS distribution, and they are 227 completely independent of the actual physical interference that 228 the network is experiencing.

229 To interpret the cell edge boundary from a physical percep230 tively, namely, linking it better to the underlying physics of 231 signal transmission medium, let us now introduce the following 232 definition that formally defines the cell edge boundary.

233 Definition 1: The cell edge boundary is constituted by the 234 group of points where the strength of the desired signal received 235 from the serving BS equals to the interfering signal's strength.

236 In other words, at the cell edge boundary, the desired signal237 to-interference ratio (SIR) is equal to 1 . This definition of cell

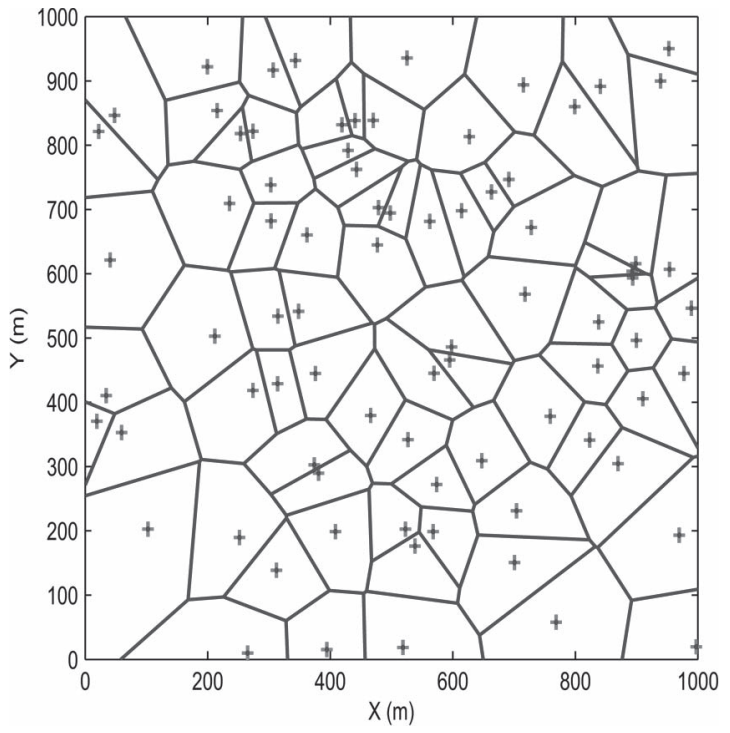

Fig. 1. Random distribution of the BSs marked by + , with the cell boundaries corresponding to a Voronoi tessellation.

edge boundary is both intuitive and practical since, within the 238 coverage area of a BS, the desired signal should be stronger than 239 the interfering signal, yielding SIR $>1$. Let us denote the $i$ th 240 BS location as the point $z_{i}$, where $i \in \Phi$. Furthermore, denote 241 the distance from $z_{i}$ to a point $z$ as $\left|z-z_{i}\right|$. The desired signal 242 power at the point $z$ provided by the $i$ th $\mathrm{BS}$ is given by

$$
\mathrm{S}(z)=\mathbb{E}\left[P h_{i}\left|z-z_{i}\right|^{-\alpha}\right]=P \bar{h}\left|z-z_{i}\right|^{-\alpha}
$$

while the interfering signal's power at $z$ is given by

$$
\begin{aligned}
\mathrm{I}(z) & =\mathbb{E}\left[I_{r}(z)\right]=\mathbb{E}\left[\sum_{j \in \Phi \backslash i} P h_{j}\left|z-z_{j}\right|^{-\alpha}\right] \\
& =P \bar{h} \sum_{j \in \Phi \backslash i}\left|z-z_{j}\right|^{-\alpha} .
\end{aligned}
$$

Thus, with respect to the $i$ th BS, the SIR at the point $z$ is 245 given by

$$
\operatorname{SIR}(z)=\frac{\left|z-z_{i}\right|^{-\alpha}}{\sum_{j \in \Phi \backslash i}\left|z-z_{j}\right|^{-\alpha}} .
$$

Therefore, at the $i$ th cell's edge boundary, we have $\operatorname{SIR}(z)=1.247$

In Figs. 2 and 3, the distribution of the BSs is based on the 248 same regular grid network model, and the number of BSs is 33. 249 As shown in Fig. 2, the shape of each cell in the network is 250 approximately a regular circle given the path-loss exponent of 251 $\alpha=3$. By contrast, observe in Fig. 3 that the cell shape changes 252 into a hexagonal one when the path-loss exponent is increased 253 to $\alpha=10$.

In Figs. 4 and 5, the locations of the 33 BSs are randomly 255 drawn from the uniform distribution across the entire network 256 area. The cells now approximately have irregularly circular 257 shapes when the path-loss exponent is $\alpha=3$, but interestingly, 258 it is the Voronoi tessellation that naturally emerges when the 259 path-loss exponent is increased to $\alpha=10$. 


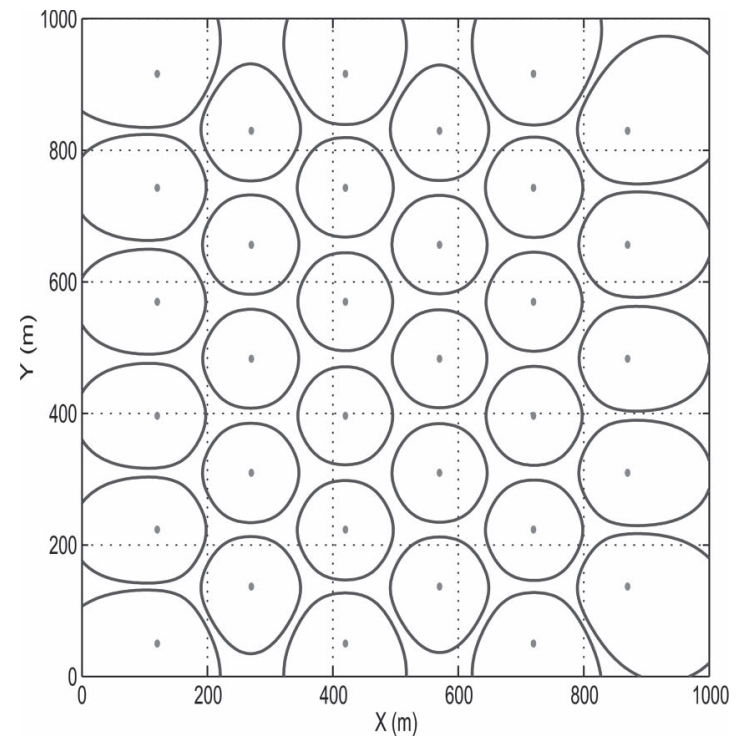

Fig. 2. Cell edge boundaries of the grid network model with the 33 BS locations marked by dots, as determined by $\operatorname{SIR}(z)=1$. The path-loss exponent is $\alpha=3$.

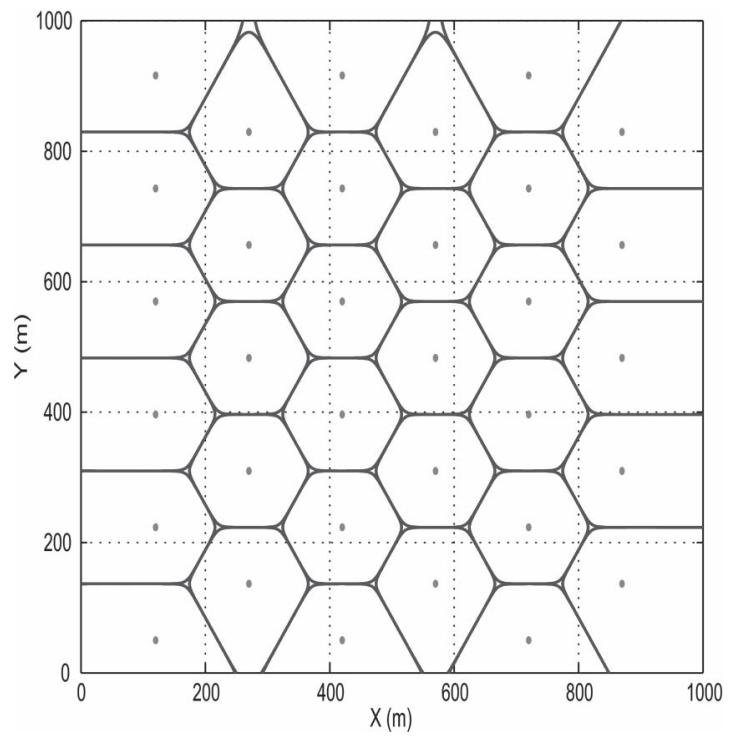

Fig. 3. Cell edge boundaries of the grid network model with the 33 BS locations marked by dots, as determined by $\operatorname{SIR}(z)=1$. The path-loss exponent is $\alpha=10$.

261 The given results demonstrate that our Definition 1 of cell 262 edge boundary is a physically plausible one for analyzing the 263 network, and both the hexagonal topology and the Voronoi 264 tessellation naturally emerge according to this definition, de265 pending on whether the geographic distribution of BSs is deter266 ministic or random and providing that the path-loss exponent 267 is sufficiently high. Note that such a high path-loss exponent 268 is unrealistic in real transmission environments. Therefore, 269 our analysis of cell edge boundary reveals a weakness of the 270 popular hexagonal and Voronoi network topologies, namely, 271 they do not reflect the underlying signal transmission medium. 272 Significantly, given a more realistic path-loss exponent of ap273 proximately three, the cell edge boundary exhibits irregular

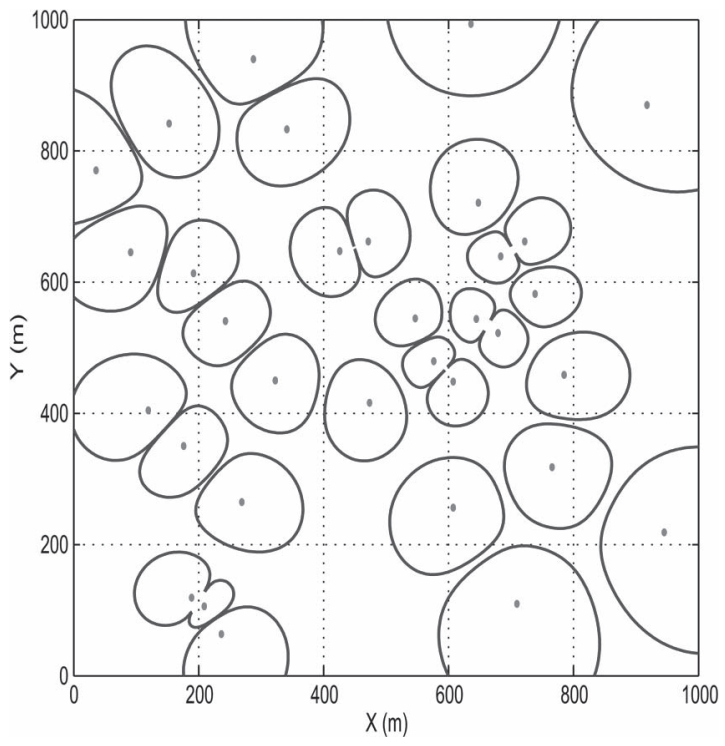

Fig. 4. Cell edge boundaries of the randomly distributed network model with the 33 BS locations marked by dots, as determined by $\operatorname{SIR}(z)=1$. The pathloss exponent is $\alpha=3$.

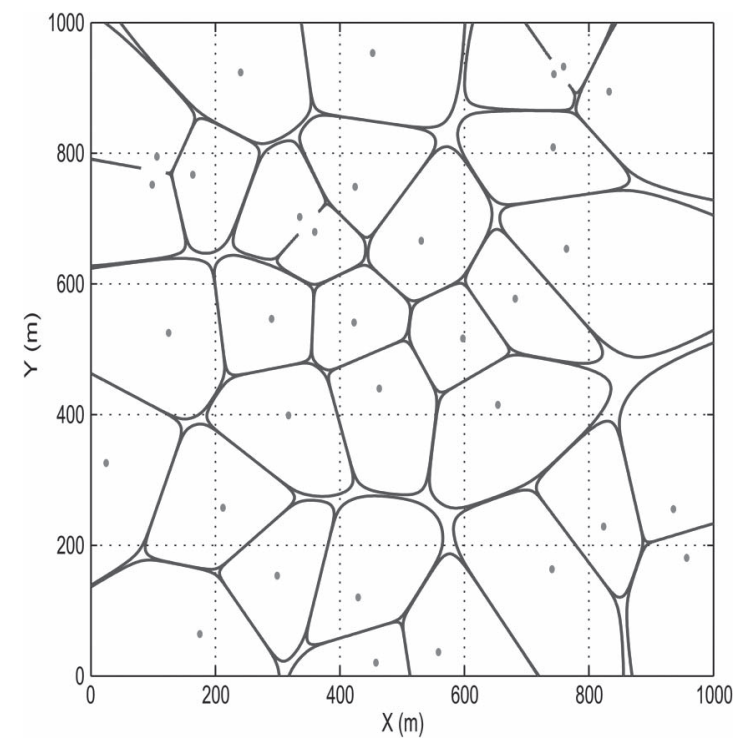

Fig. 5. Cell edge boundaries of the randomly distributed network model with the 33 BS locations marked by dots, as determined by $\operatorname{SIR}(z)=1$. The pathloss exponent is $\alpha=10$.

near-circular cell shapes, for which neither hexagonal topology 274 nor Voronoi tessellation can be used to accurately model. 275 Furthermore, the "weak" coverage areas that are left outside 276 any cell boundary, where the desired signal is weaker than the 277 interfering signals, as shown in Fig. 4, highlight the benefits of 278 employing collaborative relaying techniques.

\section{EQuivalent Uniform Density Plane-Entity FOR 280 CUMUlative INTERFERENCE CALCULATION

To accurately analyze the coverage probability and the 282 achievable rate, it is necessary to find an efficient means for 283 


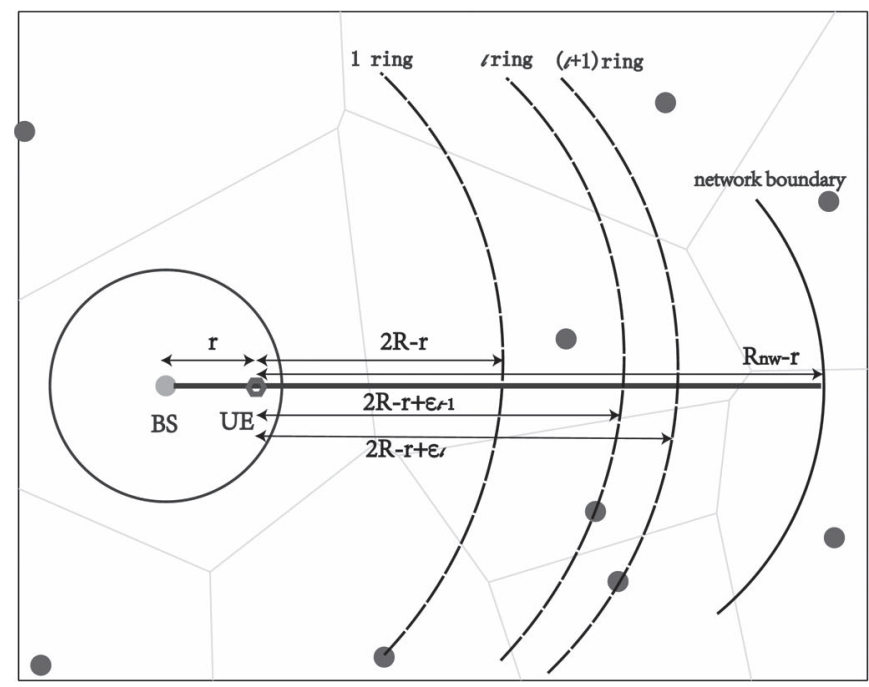

Fig. 6. Proposed EUDPE method for calculating the other-cell interference.

284 cumulative interference calculation. By considering the dis285 tribution of the interference imposed by the BSs in the law 286 of large numbers and combining it with the fluid model of 287 [26], we propose the EUDPE method for calculating the cu288 mulative interference. The basic idea of this EDUPE method 289 is as follows. Although the actual geographic distribution of 290 BSs always shows a certain degree of irregularity, we may 291 define a group of equivalent and uniformly distributed BSs for 292 approximating the other-cell CCI. Since, in real-world cellular 293 networks, the actual geographic distribution of BSs is often 294 close to a uniform random distribution, such an approximation 295 is sufficiently accurate. It is worth emphasizing however that 296 we do not assume a uniform and random BS distribution for 297 the actual network to be modeled. More specifically, given 298 a network having the average BS density of $\rho$, we approx299 imate this network with an equivalent network whose BSs 300 are uniformly distributed and whose BS density is also $\rho$. 301 Such a network is termed the equivalent EUDPE of the given 302 network. With the aid of our EUDPE method, we can calcu303 late or approximate the cumulative interference for any given 304 network.

305 Fig. 6 illustrates the concept of the EUDPE, where the 306 serving BS is assumed the origin of the polar coordinate plane. 307 Since the coverage radius of a BS is $R$, the distance between 308 two neighboring BSs is $2 R$, where $R \propto(1 / \sqrt{\rho})$. For notational 309 simplification, we drop the subscript 0 from $r_{0}$ and denote the 310 distance from the serving BS to the UE as $r$, where $0 \leq r \leq R$. 311 Thus, the distance from the nearest interfering BS to the UE 312 is $(2 R-r)$. As shown in Fig. 6, the network's coverage area 313 is partitioned by the $N_{r}$ rings, and the distance from the UE 314 to the $l$ th ring is given by $\left(2 R-r+\varepsilon_{l-1}\right)$, where $1 \leq l \leq$ $315 N_{r}$ with $\varepsilon_{0}=0$ and $\left(2 R-r+\varepsilon_{N_{r}}\right)=R_{\mathrm{nw}}-r$. The number 316 of BSs within the area between the $l$ th and $(l+1)$ th rings 317 is approximately $\int_{0}^{2 \pi} \int_{2 R-r+\varepsilon_{l-1}}^{2 R-r+\varepsilon_{l}} \rho z d z d \theta$ when assuming the 318 equivalent EUDPE having the BS density of $\rho$. Furthermore, 319 each of these equivalent BSs has the same instantaneous 320 fast fading channel power of $\widetilde{h}_{l}$, and the mean of $\widetilde{h}_{l}$ is $\bar{h}$.
Thus, the cumulative interference $I_{r}$ can be approximated 321 according to

$$
\begin{aligned}
I_{r} & =\sum_{l=1}^{N_{r}} \int_{0}^{2 \pi} \int_{2 R-r+\varepsilon_{l-1}}^{2 R-r+\varepsilon_{l}} P \widetilde{h}_{l} z^{-\alpha} \rho z d z d \theta \\
& =\sum_{l=1}^{N_{r}} \frac{2 \pi \rho P \widetilde{h}_{l}}{\alpha-2}\left(\left(2 R-r+\varepsilon_{l-1}\right)^{2-\alpha}-\left(2 R-r+\varepsilon_{l}\right)^{2-\alpha}\right) .
\end{aligned}
$$

Theorem 1: The average of $I_{r}$ is given by

$$
\mathbb{E}\left[I_{r}\right]=\frac{2 \pi \rho P \bar{h}}{\alpha-2}\left((2 R-r)^{2-\alpha}-\left(R_{\mathrm{nw}}-r\right)^{2-\alpha}\right) .
$$

Proof: According to the Campbell-Mecke theorem [27], 324 we have

$$
\begin{aligned}
\mathbb{E} & {\left[\sum_{l=1}^{N_{r}} \frac{2 \pi \rho P \widetilde{h}_{l}}{\alpha-2}\left(\left(2 R-r+\varepsilon_{l-1}\right)^{2-\alpha}-\left(2 R-r+\varepsilon_{l}\right)^{2-\alpha}\right)\right] } \\
& =\sum_{l=1}^{N_{r}} \frac{2 \pi \rho P \mathbb{E}\left[\widetilde{h}_{l}\right]}{\alpha-2}\left(\left(2 R-r+\varepsilon_{l-1}\right)^{2-\alpha}-\left(2 R-r+\varepsilon_{l}\right)^{2-\alpha}\right) \\
& =\frac{2 \pi \rho P \bar{h}}{\alpha-2}\left((2 R-r)^{2-\alpha}-\left(R_{\mathrm{nw}}-r\right)^{2-\alpha}\right) .
\end{aligned}
$$

Typically, the path-loss exponent is $\alpha>2$ in realistic net- 327 works. Noting that $\left(R_{\mathrm{nw}}-r\right)^{2-\alpha} \rightarrow 0$ as $R_{\mathrm{nw}} \rightarrow+\infty$, we 328 have the following corollary.

Corollary 1: Given that the network's boundary is suffi- 330 ciently far away, namely, $R_{\mathrm{nw}} \rightarrow+\infty$, we have

$$
\mathbb{E}\left[I_{r}\right]=\frac{2 \pi \rho P \bar{h}}{\alpha-2}(2 R-r)^{2-\alpha} .
$$

\section{Coverage Probability Analysis Using

As mentioned earlier, the cellular system interference model 334 and the BS geographic distribution model are required in cov- 335 erage analysis. Our proposed EUDPE is a universal method 336 for evaluating the other-cell interference for all existing BS 337 distribution models, such as the stochastic PD and UD BS 338 models and the deterministic grid-based model.

\section{A. Coverage Probability Analysis Using EUDPE-PD}

Since a popular geographic BS distribution is the Poisson 341 distribution [12]-[15], we first consider the PD BS model. The 342 probability density function (pdf) of the Poisson distribution 343 can be derived using the method of [28]. Let $\lambda$ be the intensity 344 of the Poisson distribution that models the BS geographic 345 distribution and $R$ be the average coverage radius of a cell. 346 Then, the probability of having no BS that is closer than $x$ is 347 given by

$$
\mathbb{P}\{r>x\}=\mathbb{P}\{\text { No BS closer than } x\}=e^{-\lambda \pi x^{2}} .
$$


349 The corresponding cumulative distribution function (cdf) is 350 then given by

$$
\mathbb{P}\{r \leq x\}=F(x)=1-e^{-\lambda \pi x^{2}} .
$$

351 Therefore, the pdf is defined as

$$
f(r)=\frac{d F(r)}{d r}=2 \pi \lambda r e^{-\pi \lambda r^{2}} .
$$

352 Given the SINR threshold $T$, the intensity $\lambda$ and the path-loss 353 exponent $\alpha$, the coverage probability is defined as

$$
\begin{aligned}
p_{c}(T, \lambda, \alpha) & =\mathbb{E}_{r}\left[\mathbb{E}_{I_{r}}[\mathbb{P}\{\operatorname{SINR}>T\}]\right] \\
& =\int_{r>0} \mathbb{E}_{I_{r}}\left[\mathbb{P}\left\{h_{0}>P^{-1} \operatorname{Tr}^{\alpha}\left(\sigma^{2}+I_{r}\right)\right\}\right] 2 \pi \lambda r e^{-\pi \lambda r^{2}} d r
\end{aligned}
$$

354 where $\mathbb{E}_{r}[\bullet]$ denotes the expectation with respect to the random 355 variable $r$.

356 1) Lower Bound for the Probability of SINR Larger Than 357 Threshold: Noting that $h_{0}$ obeys the exponential distribution 358 with the mean $\bar{h}$, the probability of the SINR larger than the 359 threshold $T$ (averaged over the interference) is given by

$$
\begin{aligned}
\mathbb{E}_{I_{r}}\left[\mathbb{P}\left\{h_{0}>P^{-1} \operatorname{Tr}^{\alpha}\left(\sigma^{2}+I_{r}\right)\right\}\right] \\
=e^{-\bar{h} P^{-1} T r^{\alpha} \sigma^{2}} \mathbb{E}_{I_{r}}\left[e^{-\bar{h} P^{-1} T r^{\alpha} I_{r}}\right] .
\end{aligned}
$$

360 Theorem 2: A lower bound for the probability of the SINR 361 greater than the threshold $T$ is expressed as

$$
\mathbb{E}_{I_{r}}\left[\mathbb{P}\left\{h_{0}>P^{-1} \operatorname{Tr}^{\alpha}\left(\sigma^{2}+I_{r}\right)\right\}\right] \geq e^{-\bar{h} T r^{\alpha} \eta(\alpha, r)}
$$

362 where

$$
\eta(\alpha, r)=P^{-1} \sigma^{2}+\frac{2 \pi \rho \bar{h}}{\alpha-2}\left((2 R-r)^{2-\alpha}-\left(R_{\mathrm{nw}}-r\right)^{2-\alpha}\right) .
$$

363

Proof: According to Jensen's inequality [29], we have

$$
\mathbb{E}_{I_{r}}\left[e^{-\bar{h} P^{-1} T r^{\alpha} I_{r}}\right] \geq e^{-\bar{h} P^{-1} T r^{\alpha} \mathbb{E}\left[I_{r}\right]} .
$$

364 Substituting (18) into (15) and noting $\mathbb{E}\left[I_{r}\right]$ of (8) leads to (16) 365 with $\eta(\alpha, r)$ given in (17).

366 Corollary 2: Given that the network boundary is sufficiently 367 far away, namely, $R_{\mathrm{nw}} \rightarrow+\infty$

$$
\mathbb{E}_{I_{r}}\left[\mathbb{P}\left\{h_{0}>P^{-1} \operatorname{Tr}^{\alpha}\left(\sigma^{2}+I_{r}\right)\right\}\right] \geq e^{-\bar{h} T r^{\alpha} \xi(\alpha, r)}
$$

368 where

$$
\xi(\alpha, r)=P^{-1} \sigma^{2}+\frac{2 \pi \rho \bar{h}}{\alpha-2}(2 R-r)^{2-\alpha} .
$$

369 2) Lower Bound for the Coverage Probability: A lower 370 bound for the coverage probability $p_{c}(T, \lambda, \alpha)$ is given by the 371 following theorem.
Theorem 3: For the network where the BS geographic 372 distribution obeys the Poisson distribution of intensity $\lambda, 373$ a lower bound for the coverage probability $p_{c}(T, \lambda, \alpha)$ is 374 given by

$$
p_{c l}(T, \lambda, \alpha)=\pi \lambda \int_{0}^{R^{2}} e^{-\bar{h} T v^{\alpha / 2} \psi(\alpha, v)-\pi \lambda v} d v
$$

where $R$ is the coverage radius of the serving $\mathrm{BS}$, and

$$
\begin{aligned}
\psi(\alpha, v)=P^{-1} \sigma^{2}+\frac{2 \pi \rho \bar{h}}{\alpha-2}( & \left(2 R-v^{1 / 2}\right)^{2-\alpha} \\
& \left.-\left(R_{\mathrm{nw}}-v^{1 / 2}\right)^{2-\alpha}\right) .
\end{aligned}
$$

Proof: From (14) and Theorem 2, as well as noting that 377 $r \leq R$, we have

$$
p_{c l}(T, \lambda, \alpha)=\int_{0}^{R} 2 \pi \lambda r e^{-\bar{h} T r^{\alpha} \eta(\alpha, r)-\pi \lambda r^{2}} d r .
$$

By defining $r^{2}=v,(23)$ is transformed into (21) with $\psi(\alpha, v) 379$ given in (22).

Corollary 3: Given that the network boundary is sufficiently 381 far away, namely, $R_{\mathrm{nw}} \rightarrow+\infty$, a lower bound for the coverage 382 probability $p_{c}(T, \lambda, \alpha)$ is expressed as

$$
p_{c l}(T, \lambda, \alpha)=\pi \lambda \int_{0}^{R^{2}} e^{-\bar{h} T v^{\alpha / 2} \chi(\alpha, v)-\pi \lambda v} d v
$$

where

$$
\chi(\alpha, v)=P^{-1} \sigma^{2}+\frac{2 \pi \rho \bar{h}}{\alpha-2}\left(2 R-v^{1 / 2}\right)^{2-\alpha} .
$$

Remark 1: In the coverage analysis for the EUDPE-PD 385 model, the average coverage radius $R$ is related to the average 386 cell area $\mathbb{E}\left[A_{s}\right]$. Noting $R \propto \sqrt{\mathbb{E}\left[A_{s}\right]}$ and $\mathbb{E}\left[A_{s}\right]=1 / \rho$, we 387 may use

$$
R=\frac{c_{f}}{\sqrt{\rho}}
$$

where $c_{f}$ is an empirically chosen factor. For example, if the 389 average cell is defined by a square shape, we have $\mathbb{E}\left[A_{s}\right]=390$ $4 R^{2}$; therefore, we have $c_{f}=1 / 2=0.5$. On the other hand, 391 if the average coverage area is calculated according to a hexag- 392 onal one, we have $\mathbb{E}\left[A_{s}\right]=2 \sqrt{3} R^{2}$, yielding $c_{f}=1 / \sqrt{2 \sqrt{3}} \approx 393$ 0.54 , whereas for the average circle-shape cell, we have $c_{f}=394$ $1 / \sqrt{\pi} \approx 0.56$

\section{B. Coverage Probability Analysis Using EUDPE-UD}

396

For many practical cellular networks, the geographic BS 397 distribution is often close to a uniform random distribution. 398 Therefore, we next consider the UD BS model with the average 399 
400 density of BSs given by $\rho$. In this case, the corresponding cdf is 401 given by

$$
\mathbb{P}\{z \leq x\}=F(x)=\frac{x^{2}}{c_{\mathrm{nm}}^{2}} \rho, \quad 0 \leq x \leq R
$$

402 where $c_{\mathrm{nm}}^{2}$ is a normalization factor, and $R$ is the coverage 403 radius of the serving BS. Thus, the pdf is given as

$$
f(r)=\frac{2 \rho}{c_{\mathrm{nm}}^{2}} r, \quad 0 \leq r \leq R
$$

404 The normalization factor $c_{\mathrm{nm}}^{2}$ is determined as follows. Assume 405 that $E\left[A_{s}\right]=R^{2} / c_{f}^{2}$, where $c_{f}$ is defined in (26), and fur406 ther note that $E\left[A_{s}\right]=1 / \rho$. From $\int_{0}^{R} f(r) d r=1$, we obtain $407 c_{\mathrm{nm}}^{2}=c_{f}^{2}$.

408 The coverage probability is therefore defined as

$$
\begin{aligned}
p_{c}(T, \rho, \alpha) & =\mathbb{E}_{r}\left[\mathbb{E}_{I_{r}}[\mathbb{P}\{\operatorname{SINR}>T\}]\right] \\
& =\frac{\rho}{c_{f}^{2}} \int_{0}^{R} \mathbb{E}_{I_{r}}\left[\mathbb{P}\left\{h_{0}>P^{-1} \operatorname{Tr}^{\alpha}\left(\sigma^{2}+I_{r}\right)\right\}\right] 2 r d r .
\end{aligned}
$$

409 A lower bound of $\mathbb{E}_{I_{r}}\left[\mathbb{P}\left\{h_{0}>P^{-1} \operatorname{Tr}^{\alpha}\left(\sigma^{2}+I_{r}\right)\right\}\right]$ is given in 410 Theorem 2. Similar to the case of the EUDPE-PD expressed in 411 Theorem 3, therefore, a lower bound for the coverage probabil412 ity $p_{c}(T, \rho, \alpha)$ is given by the following theorem.

413 Theorem 4: For the network where the BS geographic distri414 bution obeys the uniform random distribution with an average 415 BS density of $\rho$, a lower bound for the coverage probability $416 p_{c}(T, \rho, \alpha)$ is given by

$$
p_{c l}(T, \rho, \alpha)=\frac{\rho}{c_{f}^{2}} \int_{0}^{R^{2}} e^{-\bar{h} T v^{\alpha / 2} \psi(\alpha, v)} d v
$$

417 where $\psi(\alpha, v)$ is defined in (22).

418 Corollary 4: Given that the network boundary is sufficiently 419 far away, a lower bound for the coverage probability $p_{c}(T, \rho, \alpha)$ 420 is expressed by

$$
p_{c l}(T, \rho, \alpha)=\frac{\rho}{c_{f}^{2}} \int_{0}^{R^{2}} e^{-\bar{h} T v^{\alpha / 2} \chi(\alpha, v)} d v
$$

421 where $\chi(\alpha, v)$ is defined in (25).

422 Remark 2: How to set the average coverage radius $R$ is 423 explained in Remark 1. Specifically, we may use $R=c_{f} / \sqrt{\rho}$, 424 where $c_{f}$ is an empirically chosen factor.

\section{C. Coverage Probability Analysis Using EUDPE-Grid}

426 With the aid of the EUDPE method, it is straightforward to 427 carry out the coverage probability analysis for all the traditional 428 deterministic grid-based cellular network models, such as the 429 squared and hexagonal ones. This is because the coverage 430 probability analysis using the EUDPE-Grid model is simply a 431 degenerated or special case of the EUDPE-UD-based analysis, where the density of BSs $\rho$ is identical everywhere in the net- 432 work, and every cell has the identical shape with the same area 433 $A_{s}$. Therefore, the lower bounds of the coverage probability for 434 the finite-size and infinite-size grid-based network models are 435 given in Theorem 4 and Corollary 4, respectively. Moreover, 436 choosing $R=1 /(2 \sqrt{\rho})$ corresponds to the grid-based network 437 with squared cells, whereas using $R=1 /(\sqrt{2 \sqrt{3}} \sqrt{\rho})$ is related 438 to considering the grid-based network with hexagonal cells. In 439 general, we may use $R=c_{f} / \sqrt{\rho}$ for any deterministic grid- 440 based network by choosing an appropriate value for $c_{f}$. It be- 441 comes obvious that, under the equivalent network environment 442 of the same $\rho$ and $R$ values, the coverage probability obtained 443 by the EUDPE-Grid-based analysis is identical to that obtained 444 by the EUDPE-UD-based analysis.

\section{Numerical Results for Coverage Probability}

We evaluated the coverage probability first by simulation and 447 used the simulated results as the benchmark for the comparison 448 with our theoretical analytic results. We considered two sce- 449 narios. The first case is a single-tier network constructed by 450 macrocells, obeying the uniform random BS distribution and 451 the cellular channel model described in Section II, whereas 452 the second network followed a Poisson BS distribution and 453 obeyed the same cellular channel model of Section II. Given 454 the SINR threshold $T$, the path-loss exponent $\alpha$, and the SINR 455 value, the simulated coverage probability was calculated using 456 the pseudocodes presented in Algorithm 1. In the simulation, 457 we set the number of BSs to $N_{\mathrm{BS}}=80$, the number of UEs to 458 $N_{\mathrm{UE}}=10000$, the network coverage area to Network Area $=459$ $1000 \times 1000 \mathrm{~m}^{2}$, and the number of sample simulations to 460 $N_{\max }=100$. The average density of BSs was then given as $\quad 461$

$$
\rho=\frac{N_{\mathrm{BS}}}{\text { Network Area }}\left[\mathrm{BSs} / \mathrm{m}^{2}\right] .
$$

For the Poisson distribution, its intensity was $\lambda=\rho$. We com- 462 pared our low-bound coverage probability results based on the 463 EUDPE-PD and EUDPE-UD models with that of the PPP- 464 based analysis [12]. Since the PPP method can only consider 465 the case of an infinitely large network, we assumed the network 466 boundary $R_{\mathrm{nw}} \rightarrow+\infty$. In the following comparison, the simu- 467 lation results obtained by the network with the uniform random 468 BS distribution are labeled as Simulated data 1, whereas the 469 simulation results yielded by the network with the Poisson BS 470 distribution are denoted Simulated data 2.
Algorithm 1 Network Simulation to Evaluate the Coverage Probability.

1: Give the number of BSs $N_{\mathrm{BS}}$, the Network Area, and the 472 number of UEs $N_{\mathrm{UE}}$;

2: Give the maximum number of sample simulations $N_{\max } ; \quad 474$

3: Set Average Coverage Probability $=0 ;$

4: for $N_{\mathrm{sm}}=1$ to $N_{\max }$ do 476

5: Uniformly and randomly draw the $N_{\mathrm{BS}}$ BSs over Net- 477 work Area, or draw the $N_{\mathrm{BS}}$ BSs over Network Area by 478 the Poisson distribution; 


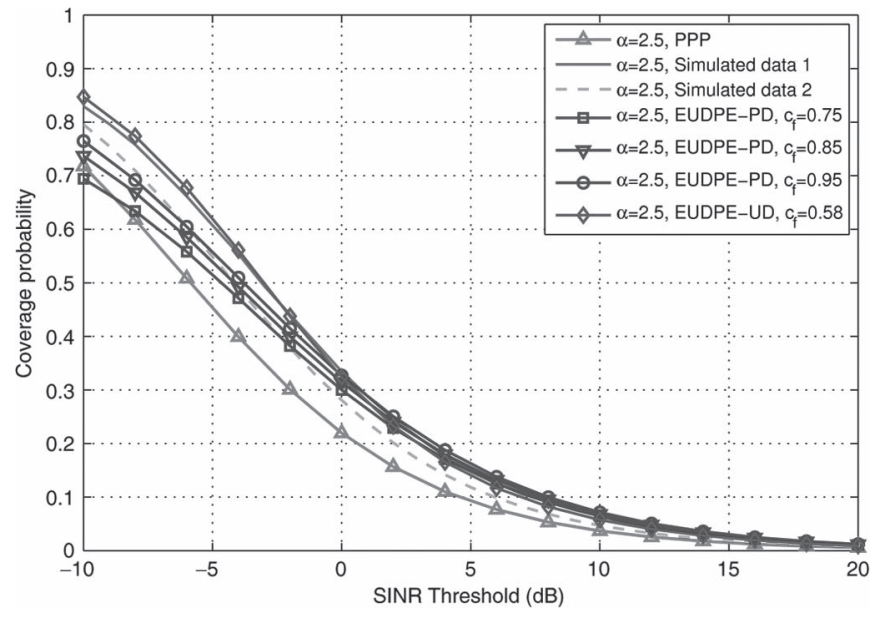

Fig. 7. Comparison of the coverage probabilities based on three different models to the network simulation results, given the path-loss exponent of $\alpha=2.5$ and no noise, i.e., the AWGN power $\sigma^{2}=0$ and SINR $=$ SIR.

6: Uniformly and randomly draw the $N_{\mathrm{UE}}$ UEs over Network Area;

482 7: Initialization: count $=0$;

483 8: for $j=1$ to $N_{\mathrm{UE}}$, do

484 9: $\quad$ if $\operatorname{SINR}_{j} \geq T$ then

485 10: $\quad$ count $=$ count +1 ;

486 11: end if

487 12: end for

488 13: Coverage Probability $=$ count $/ N_{\mathrm{UE}}$;

489 14: Average Coverage Probability $+=$

$490 \quad$ Coverage Probability;

\section{15: end for}

492 16: Average Coverage Probability / $=N_{\max }$.

493 Given the path-loss exponent of $\alpha=2.5$ and assuming no 494 AWGN or $\sigma^{2}=0$, which implies SINR $=$ SIR, Fig. 7 shows 495 the coverage probabilities calculated based on the three analytic 496 models, in comparison to the coverage probabilities obtained by 497 the two different network simulations, when varying the SINR 498 threshold. It is shown in Fig. 7 that the coverage probability 499 analysis results of our proposed EUDPE-PD and EUDPE-UD 500 models agree with both simulation results well, better than the 501 PPP-based analysis. When the path-loss exponent is increased 502 to $\alpha=3$ and 4 , the results obtained are shown in Figs. 8 503 and 9, respectively, where it can be seen that the EUDPE504 UD analysis agrees with the simulation result based on the 505 network with the uniform random BS distribution better than 506 the other two models, whereas the PPP-based analysis agrees 507 better with the simulation result of the network with the Poisson 508 BS distribution better than the other two models.

509 It is worth emphasizing that because there exist no real 510 network performance data to validate an analysis model, we 511 can only rely on the simulated data. When we have an analysis 512 model agrees with a particular simulation result better than an513 other analysis model, it does not imply that the former is better 514 than the latter. The particular simulation result may not actually 515 represent the true real network performance and, moreover, the 516 simulation conditions may not actually match those imposed 517 on an analysis model. What we can claim however is that, if

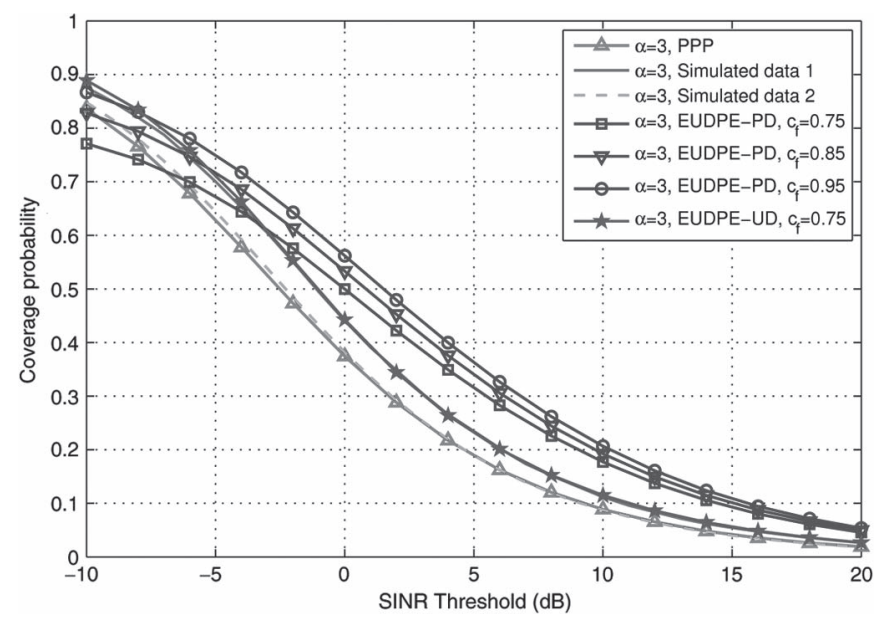

Fig. 8. Comparison of the coverage probabilities based on three different models to the network simulation results, given the path-loss exponent of $\alpha=3$ and no noise, i.e., the AWGN power $\sigma^{2}=0$ and SINR $=$ SIR.

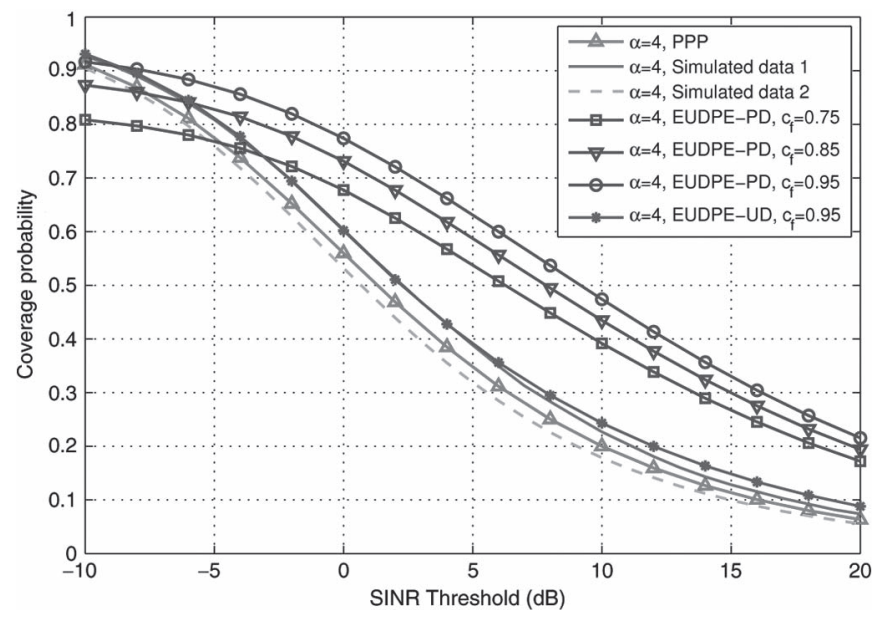

Fig. 9. Comparison of the coverage probabilities based on three different models to the network simulation results, given the path-loss exponent of $\alpha=4$ and no noise, i.e., the AWGN power $\sigma^{2}=0$ and SINR $=$ SIR.

an analysis model agrees well with simulation data, it is a rea- 518 sonable tool for network analysis and planning. Similarly, if a 519 lower bound coverage probability derived by an analysis model 520 appears to be larger than a simulated coverage probability, it 521 does not imply that this analysis model is wrong. Again, the 522 simulation conditions may not actually match those imposed 523 on the analysis model. For example, we assumed that the 524 network boundary $R_{\mathrm{nw}} \rightarrow+\infty$ for the proposed EUDPE-PD 525 and EUDPE-UD models and the PPP-based analysis for the fair 526 comparison of the three analysis models since the PPP method 527 can only be applied for the case of an infinitely large network. 528 However, the simulated network size was $1000 \times 1000 \mathrm{~m}^{2}$ and 529 not infinitely large. As shown earlier, another advantage of 530 our analysis approach over the PPP-based method is that our 531 method can be applied to analyze finite-size networks.

In our EUDPE-based analysis, the empirical chosen factor 533 $c_{f}$ is related to the average cell shape and size. The theoretical 534 explanations of this area factor $c_{f}$ are given in Remark 1.535 Observe from Fig. 7 that, for the path-loss exponent $\alpha=2.5$, an 536 appropriate value of this area factor for our EUDPE-UD model 537 is $c_{f}=0.58$, which is, in fact, close to the case of the average 538 


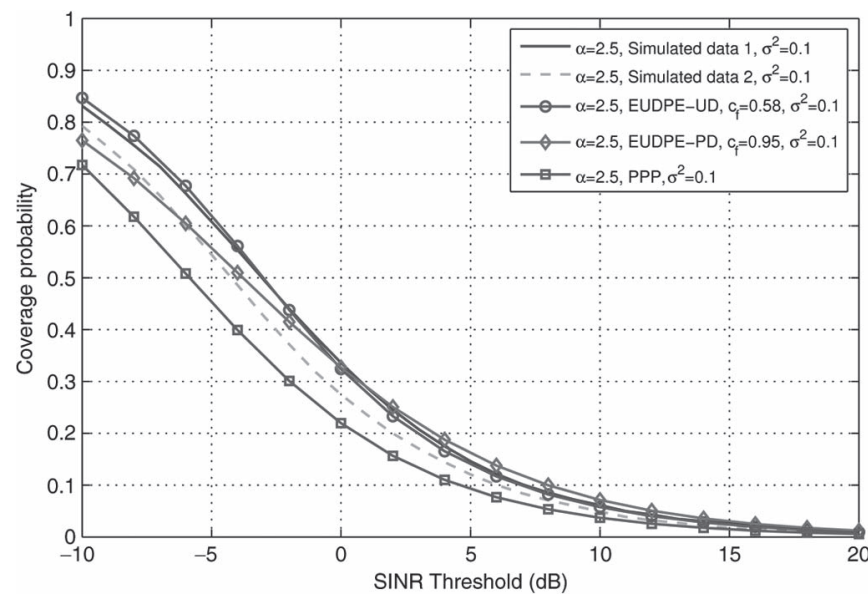

Fig. 10. Comparison of the coverage probabilities based on three different models to the network simulation results, given the path-loss exponent of $\alpha=$ 2.5 and the AWGN power $\sigma^{2}=0.1$.

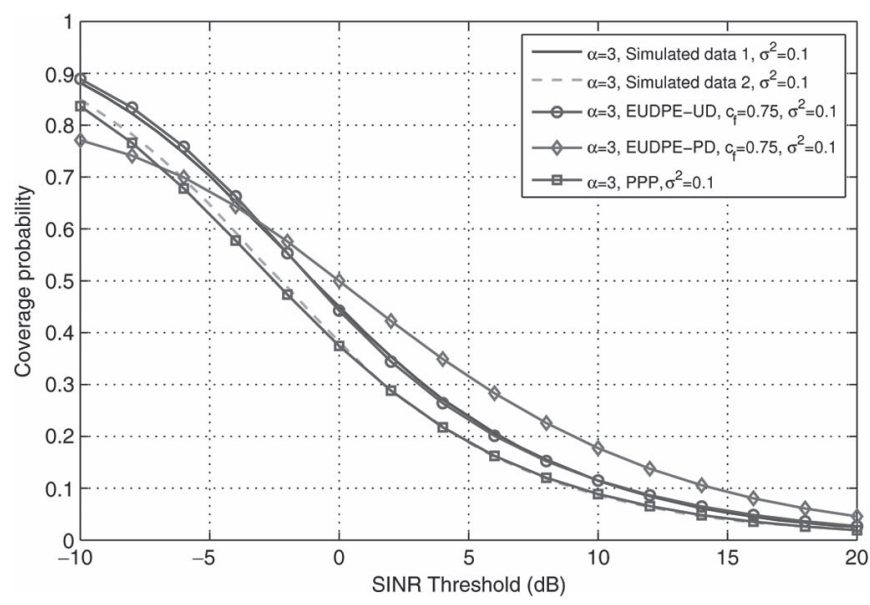

Fig. 11. Comparison of the coverage probabilities based on three different models to the network simulation results, given the path-loss exponent of $\alpha=3$ and the AWGN power $\sigma^{2}=0.1$.

539 circle-shaped cell. However, as shown in Figs. 8 and 9, as $\alpha$ 540 increases, the appropriate area factor $c_{f}$ value also increases. A 541 plausible explanation for this phenomenon is offered as follows. 542 As the path-loss exponent $\alpha$ increases, the effective coverage 543 area $R^{2} / c_{f}^{2}$ of the serving BS is reduced, and this corresponds 544 to an increase in the area factor $c_{f}$.

545 Next, the effect of noise imposed on the achievable coverage 546 probability was investigated by setting the AWGN power to $547 \sigma^{2}=0.1$ or $10 \log _{10}\left(1 / \sigma^{2}\right)=10 \mathrm{~dB}$, and the results obtained 548 are given in Figs. 10-12, respectively, for the three differ549 ent values of $\alpha$. For graphic clarity, we only draw a single 550 EUDPE-PD-based coverage probability associated with an ap551 propriate area factor $c_{f}$ value in each of these three figures. 552 Again, the same observations as those drawn for Figs. 7-9 can 553 be made, namely, for the case of $\alpha=2.5$, the EUDPE-UD554 based analysis agrees with the both simulation results better 555 than the PPP-based analysis, whereas for higher $\alpha$ values, the 556 EUDPE-UD analysis matches better with the simulated results 557 based on the uniform random BS distribution, and the PPP558 based analysis agrees better with the simulated results based

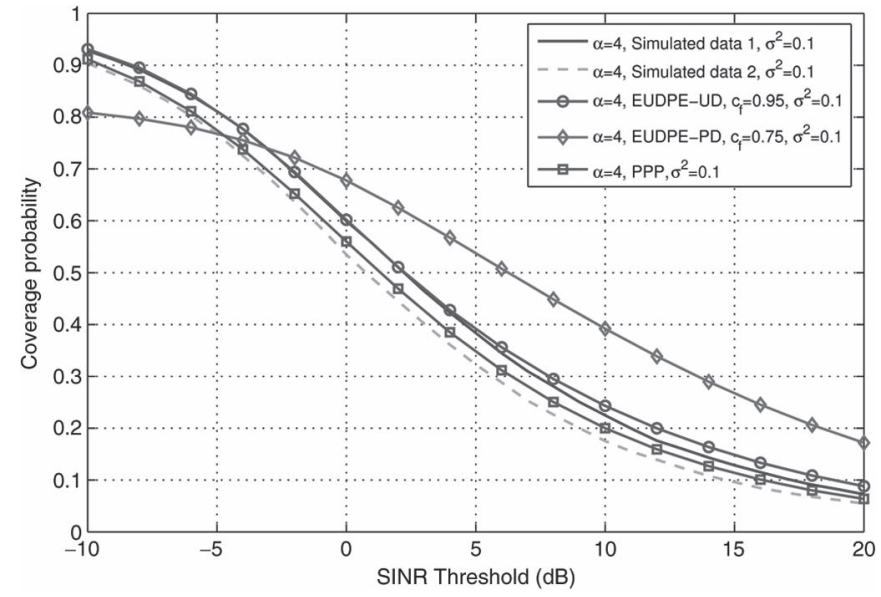

Fig. 12. Comparison of the coverage probabilities based on three different models to the network simulation results, given the path-loss exponent of $\alpha=4$ and the AWGN power $\sigma^{2}=0.1$.

on the Poisson BS distribution. Upon comparing Figs. 10-12 559 with Figs. 7-9, it can be seen that the effect of the channel 560 AWGN to the achievable coverage probability is minor. For 561 example, observe that the simulated-data-2 curve in Fig. 7562 almost matches the simulated-data-2 curve in Fig. 10, whereas 563 the PPP-analysis-based curve in Fig. 7 is almost identical to the 564 PPP-analysis-based curve in Fig. 10. Similarly, the other three 565 coverage probability curves in Fig. 10 also closely match the 566 corresponding coverage probability curves in Fig. 7.

\section{Average Achievable Rate Analysis Using EQUiVALENT UNIFORM DENSITY Plane-ENTITY}

Let us now apply the proposed EUDPE method to analyze 570 the average achievable throughput. According to Shannon's 571 theory, under the idealized simplifying condition of having a 572 Gaussian interference owing to the central limit theorem, the 573 average achievable rate is defined as [12]

$$
C \triangleq \mathbb{E}[\ln (1+\operatorname{SINR})] .
$$

Since we are concerned with the system's achievable through- 575 put, we will consider the case of the network boundary being 576 sufficiently far away, i.e., $R_{\mathrm{nw}} \rightarrow+\infty$.

\section{A. Average Achievable Rate Analysis Using EUDPE-PD}

Again, we first consider the case that the geographic BS 579 distribution follows a Poisson distribution, and we have the 580 following result.

Theorem 5: For the network where the BS geographic 582 distribution obeys the Poisson distribution of intensity $\lambda$, a 583 lower bound for the average achievable throughput is given by 584

$$
C_{l}(\lambda, \alpha)=\pi \lambda \int_{0}^{R^{2}} e^{-\pi \lambda v}\left(\int_{t>0} e^{-\bar{h} v^{\alpha / 2}\left(e^{t}-1\right) \chi(\alpha, v)} d t\right) d v
$$

where $\chi(\alpha, v)$ is given in (25). 


$$
\begin{aligned}
& C(\lambda, \alpha)=\int_{0}^{R} 2 \pi \lambda r e^{-\pi \lambda r^{2}} \\
& \times \int_{t>0} \mathbb{E}_{I_{r}}\left[\mathbb{P}\left\{h_{0}>P^{-1} r^{\alpha}\left(e^{t}-1\right)\left(\sigma^{2}+I_{r}\right)\right\}\right] d t d r .
\end{aligned}
$$

587 Similar to Corollary 2, we have

$$
\begin{aligned}
\mathbb{E}_{I_{r}}\left[\mathbb { P } \left\{h_{0}>P^{-1} r^{\alpha}\left(e^{t}-1\right)\left(\sigma^{2}\right.\right.\right. & \left.\left.\left.+I_{r}\right)\right\}\right] \\
& \geq e^{-\bar{h} r^{\alpha}\left(e^{t}-1\right) \xi(\alpha, r)}
\end{aligned}
$$

588 where $\xi(\alpha, r)$ is defined in (20). Thus, a lower bound of $C(\lambda, \alpha)$ 589 is given by

$$
C_{l}(\lambda, \alpha)=\int_{0}^{R} 2 \pi \lambda r e^{-\pi \lambda r^{2}}\left(\int_{t>0} e^{-\bar{h} r^{\alpha}\left(e^{t}-1\right) \xi(\alpha, r)} d t\right) d r .
$$

590 By defining $v=r^{2}$ in (37), we obtain (34).

591 Corollary 5: In the noise-free case, namely, $\sigma^{2}=0$, a lower 592 bound for the average achievable throughput is

$$
C_{l}(\lambda, \alpha)=\pi \lambda \int_{0}^{R^{2}} e^{-\pi \lambda v}\left(\int_{t>0} e^{-\bar{h} v^{\alpha / 2}\left(e^{t}-1\right) \bar{\chi}(\alpha, v)} d t\right) d v
$$

593 where

$$
\bar{\chi}(\alpha, v)=\frac{2 \pi \rho \bar{h}}{\alpha-2}\left(2 R-v^{1 / 2}\right)^{2-\alpha} .
$$

\section{B. Average Achievable Rate Analysis Using EUDPE-UD}

595 Next, we consider the case that the geographic BS distribu596 tion follows a uniform random distribution, and we have the 597 following result.

598 Theorem 6: For the network where the BS geographic dis599 tribution obeys the uniform random distribution with an average 600 BS density of $\rho$, a lower bound for the average achievable 601 throughput is given by

$$
C_{l}(\rho, \alpha)=\frac{\rho}{c_{f}^{2}} \int_{0}^{R^{2}}\left(\int_{t>0} e^{-\bar{h} v^{\alpha / 2}\left(e^{t}-1\right) \chi(\alpha, v)} d t\right) d v
$$

602 where $\chi(\alpha, v)$ is given in (25).

603 Proof: Noting that the average achievable throughput is 604 defined as

$$
\begin{aligned}
& C(\lambda, \alpha)=\frac{\rho}{c_{f}^{2}} \int_{0}^{R} 2 r \\
& \times \int_{t>0} \mathbb{E}_{I_{r}}\left[\mathbb{P}\left\{h_{0}>P^{-1} r^{\alpha}\left(e^{t}-1\right)\left(\sigma^{2}+I_{r}\right)\right\}\right] d t d r
\end{aligned}
$$

605 the proofs are similar to the proofs for Theorem 5 .
Corollary 6: In the noise-free case, namely, $\sigma^{2}=0$, a lower 606 bound for the average achievable throughput is

$$
C_{l}(\rho, \alpha)=\frac{\rho}{c_{f}^{2}} \int_{0}^{R^{2}}\left(\int_{t>0} e^{-\bar{h} v^{\alpha / 2}\left(e^{t}-1\right) \bar{\chi}(\alpha, v)} d t\right) d v .
$$

where $\bar{\chi}(\alpha, v)$ is given in (39).

608

Remark 3: It is straightforward to carry out the average 609 achievable throughput analysis for any deterministic grid-based 610 cellular network model, because the EUDPE-Grid model is a 611 special case of the EUDPE-UD model. Therefore, the lower 612 bound of the average achievable throughput for the grid-based 613 network model is also given in Theorem 6. Moreover, under the 614 equivalent network environment of the same $\rho$ and $R$ values, 615 the lower bound of the average achievable throughput obtained 616 by the EUDPE-Grid-based analysis is identical to that obtained 617 by the EUDPE-UD-based analysis.

\section{Numerical Results for Average Achievable Rate}

619

Assuming a unity frequency reuse factor, we compare the 620 lower bounds of the average achievable throughput obtained 621 by the proposed EUDPE-PD- and EUDPE-UD-based analyses 622 to that of the PPP-based analysis [12] in Fig. 13 by varying 623 the path-loss exponent value. The simulated average achiev- 624 able throughputs obtained from the two network simulations 625 with the uniform random BS distribution and the Poisson BS 626 distribution are labeled as Simulated rate 1 and Simulated 627 rate 2, respectively, and they are also given in Fig. 13 as the 628 benchmark. For our proposed EUDPE-PD and EUDPE-UD- 629 based analysis and the network simulations, both the noise- 630 free and noisy results are presented. However, for the 631 PPP-based average achievable throughput analysis, only the 632 noise-free case is provided in [12]; therefore, in Fig. 13, we only 633 present the noise-free PPP-based result. It can be observed that 634 all the three theoretical analysis based results and the simulation 635 data all reveal that the average achievable throughput increases 636 linearly, as the path-loss exponent increases. More specifically, 637 all the analytical and simulated data have accurate linear fitting. 638 It is also shown in Fig. 13 that our proposed EUDPE-PD- 639 and EUDPE-UD-based analyses agree with the two simulated 640 results better than the PPP-based analysis, particularly for the 641 path-loss exponent $\alpha \leq 4.5$. The results of Fig. 13 also show 642 that the noise only has a minor effect on the average achievable 643 throughput, which is expected as we consider the interference- 644 limited scenario with a unity frequency reuse factor.

\section{CONCLUSION}

646

We have proposed a universal approach for accurately 647 analyzing the coverage probability and average achievable 648 throughput of cellular networks. More specifically, we have 649 derived a generic EUDPE procedure for evaluating the other- 650 cell interference. Based on this EUDPE interference model, we 651 have derived the lower bounds of both the coverage probability 652 and average achievable throughput for various practical BS 653 distribution models, including the stochastic Poisson distributed 654 model, uniformly and randomly distributed model, and the 655 


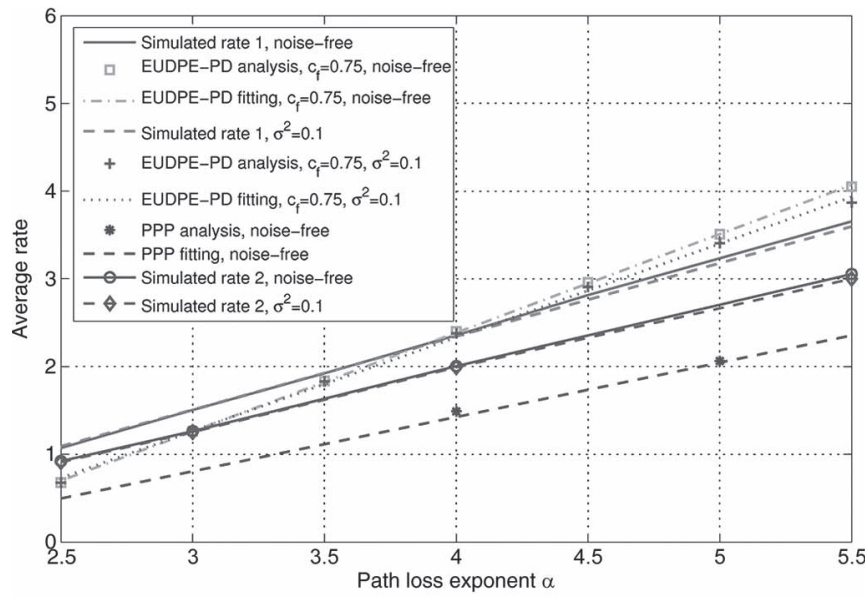

(a)

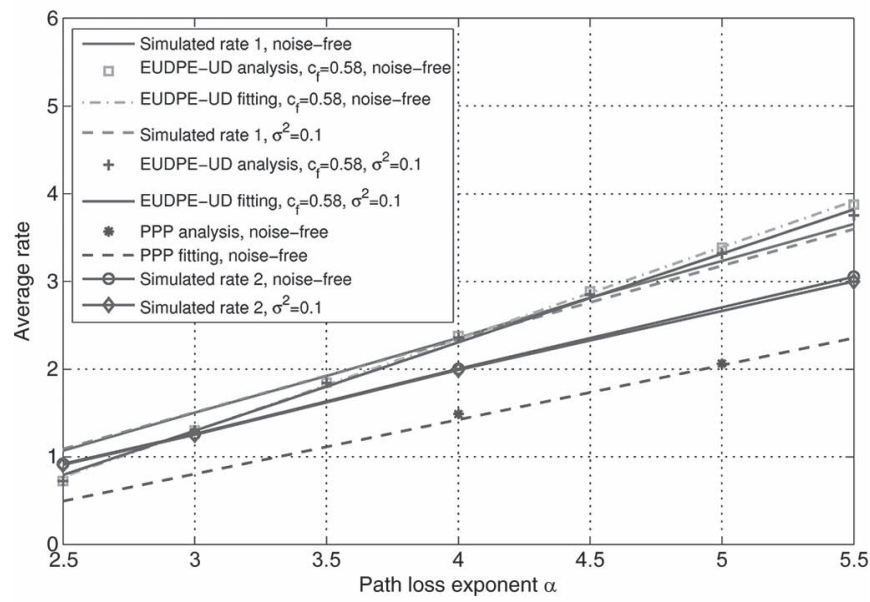

(b)

Fig. 13. Comparison of the average achievable throughputs based on three different models to the network simulation results, given different path-loss exponent values. (a) EUDPE-PD and PPP models and (b) EUDPE-UD and PPP models.

656 deterministic grid-based model. Extensive simulation results 657 have validated that the coverage probability and average 658 throughput obtained by our proposed universal analysis method 659 agree with the simulated coverage probability and average 660 throughput at least as closely as those obtained by the popular 661 existing PPP-based analysis, if not better. In addition, we have 662 also introduced a generic and physical definition of cell edge 663 boundary. We have shown that the popular hexagonal and 664 Voronoi network topologies only emerge from the grid-based 665 network model and the random BS distribution model, respec666 tively, given an unrealistic high path-loss exponent according 667 to this definition. Moreover, we have demonstrated that the cell 668 edge boundary shows irregular near-circular shapes, given a 669 more realistic path-loss exponent, which cannot be modeled 670 accurately by either hexagonal or Voronoi topology.

\section{REFERENCES}

1] A. Damnjanovic et al., "A survey on 3GPP heterogeneous networks," IEEE Wireless Commun., vol. 18, no. 3, pp. 10-21, Jun. 2011.

[2] J. G. Andrews, "Seven ways that hetnets are a cellular paradigm shift," IEEE Commun. Mag., vol. 51, no. 3, pp. 136-144, Mar. 2013.

[3] A. Ghosh, et al., "Heterogeneous cellular networks: From theory to practice," IEEE Commun. Mag., vol. 50, no. 6, pp. 54-64, Jun. 2012.
[4] J. G. Andrews, H. Claussen, M. Dohler, S. Rangan, and M. C. Reed, 678 "Femtocells: Past, present, and future," IEEE J. Sel. Areas Commun., 679 vol. 30, no. 3, pp. 497-508, Apr. 2012.

[5] X. Yang and A. P. Petropulu, "Co-channel interference modeling and 681 analysis in a Poisson field of interferers in wireless communications," 682 IEEE Trans. Signal Process., vol. 51, no. 1, pp. 64-76, Jan. 2003.

[6] A. D. Wyner, "Shannon-theoretic approach to a Gaussian cellular 684 multiple-access channel," IEEE Trans. Inf. Theory, vol. 40, no. 6, 685 pp. 1713-1727, Nov. 1994

686

[7] S. Shamai and A. D. Wyner, "Information-theoretic considerations for 687 symmetric, cellular, multiple-access fading channels, Part I," IEEE Trans. 688 Inf. Theory, vol. 43, no. 6, pp. 1877-1894, Nov. 1997.

[8] S. Shamai and A. D. Wyner, "Information-theoretic considerations 690 for symmetric, cellular, multiple-access fading channels, Part II," IEEE 691 Trans. Inf. Theory, vol. 43, no. 6, pp. 1895-1911, Nov. 1997.

[9] J. Xu, J. Zhang, and J. G. Andrews, "On the accuracy of the Wyner 693 model in cellular networks," IEEE Trans. Wireless Commun., vol. 10, 694 no. 9, pp. 3098-3109, Sep. 2011.

695

[10] F. Baccelli, M. Klein, M. Lebourges, and S. Zuyev, "Stochastic 696 geometry and architecture of communication networks," Telecommun. 697 Syst., vol. 7, no. 1-3, pp. 209-227, Jun. 1997.

698

[11] T. X. Brown, "Cellular performance bounds via shotgun cellular 699 systems," IEEE J. Sel. Areas Commun., vol. 18, no. 11, pp. 2443-2455, 700 Nov. 2000.

701

[12] J. G. Andrews, F. Baccelli, and R. K. Ganti, "A tractable approach to 702 coverage and rate in cellular networks," IEEE Trans. Commun., vol. 59, 703 no. 11, pp. 3122-3134, Nov. 2011.

704

[13] R. W. Heath and M. Kountouris, "Modeling heterogeneous network 705 interference," in Proc. IEEE ITA, San Diego, CA, USA, Feb. 5-10, 2012, 706 pp. 17-22.

707

[14] H. S. Dhillon, R. K. Ganti, F. Baccelli, and J. G. Andrews, "Modeling 708 and analysis of K-tier downlink heterogeneous cellular networks," IEEE 709 J. Sel. Areas Commun., vol. 30, no. 3, pp. 550-560, Apr. 2012.

[15] M. Z. Win, P. C. Pinto, and L. A. Shepp, "A mathematical theory 711 of network interference and its applications," Proc. IEEE, vol. 97, no. 2, 712 pp. 205-230, Feb. 2009.

713

[16] A. Busson and G. Chelius, "Point processes for interference modeling 714 in CSMA/CA ad-hoc networks," in Proc. 6th ACM Symp. Performance 715 Eval. Wireless Ad Hoc, Sens., Ubiquitous Netw., Tenerife, Canary Islands, 716 Spain, Oct. 26-30, 2009, pp. 33-40.

717

[17] F. Baccelli, B. Błaszczyszyn, and P. Mühlethaler, "An Aloha protocol 718 for multihop mobile wireless networks," IEEE Trans. Inf. Theory, vol. 52, 719 no. 2, pp. 421-436, Feb. 2006

720

[18] H. Q. Nguyen, F. Baccelli, and D. Kofman, "A stochastic geometry 721 analysis of dense IEEE 802.11 networks," in Proc. 26th IEEE INFOCOM, 722 Anchorage, AK, USA, May 6-12, 2007, pp. 1199-1207.

[19] M. L. Huber and R. L. Wolpert, "Likelihood-based inference for Matérn 724 type-III repulsive point processes," Adv. Appl. Probab., vol. 41, no. 4, 725 pp. 958-977, Dec. 2009.

726

[20] M. Haenggi, "Mean interference in hard-core wireless networks," IEEE 727 Commun. Lett., vol. 15, no. 8, pp. 792-794, Aug. 2011.

728

[21] A. Hasan and J. G. Andrews, "The guard zone in wireless ad hoc 729 networks," IEEE Trans. Wireless Commun., vol. 6, no. 3, pp. 897-906, 730 Mar. 2007.

731

[22] B. Cho, K. Koufos, and R. Jantti, "Bounding the mean interference 732 in Matérn type II hard-core wireless networks," IEEE Wireless Commun. 733 Lett., vol. 2, no. 5, pp. 563-566, Oct. 2013.

[23] F. Jarai-Szabo and Z. Neda, "On the size-distribution of Poisson 735 Voronoi cells," Phys. A, Statist. Mech. Appl., vol. 385, no. 2, pp. 518-526, 736 Feb. 2007.

[24] D. Cao, S. Zhou, and Z. Niu, "Optimal combination of base sta- 738 tion densities for energy-efficient two-tier heterogeneous cellular net- 739 works," IEEE Trans. Wireless Commun., vol. 12, no. 9, pp. 4350-4362, 740 Sep. 2013.

[25] S. Lee and K. Huang, "Coverage and economy of cellular networks with 742 many base stations," IEEE Commun. Lett., vol. 16, no. 7, pp. 1038-1040, 743 Jul. 2012

744

[26] J.-M. Kelif, M. Coupechoux, and P. Godlewski, "A fluid model for per- 745 formance analysis in cellular networks," EURASIP J. Wireless Commun. 746 Netw., vol. 2010, pp. 1-11, Aug. 2010.

[27] F. Baccelli and B. Blaszczyszyn, Stochastic Geometry and Wireless 748 Networks, Volume I: Theory. Hanover, MA, USA: Now, 2009.

[28] M. Haenggi, Stochastic Geometry for Wireless Networks, Cambridge, 750 U.K.: Cambridge Univ. Press, 2013.

[29] M. Kuczma, An Introduction to the Theory of Functional Equations 752 and Inequalities: Cauchy's Equation and Jensen's Inequality. Basel, 753 Switzerland: Birkhauser, 2008. 


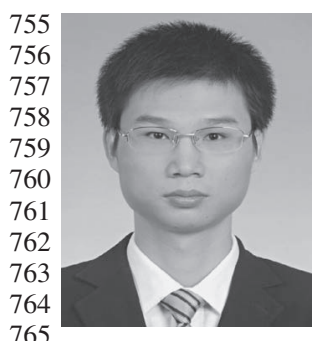

Hui Zhang received the B.Eng. degree in applied mathematics and the Ph.D. degree in electrical engineering from Beijing University of Posts and Telecommunications, Beijing, China, in 2005 and 2010 , respectively.

Since 2010, he has been a faculty member with the School of Electrical Information and Optical Engineering, Nankai University, Nankai, China. From 2013 to 2014, he was a Postdoctoral Scholar with the School of Electrical and Computer Science, University of Southampton, Southampton, U.K. In 2014, he 766 joined in the China-Korea Young Scientist Exchange Program. His research 767 interests include cellular networks and wireless communication theory.

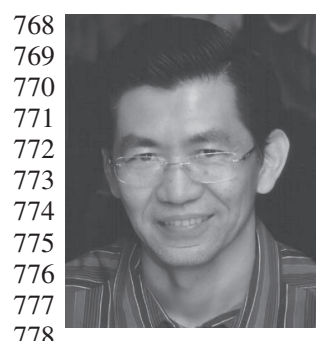

Sheng Chen (M'90-SM'97-F'08) received the B.Eng. degree in control engineering from the East China Petroleum Institute, Dongying, China, in 1982; the Ph.D. degree in control engineering from the City University London, London, U.K., in 1986; and the D.Sc. degree from the University of Southampton, Southampton, U.K., in 2005.

From 1986 to 1999 , he held research and academic appointments with The University of Sheffield, Sheffield, U.K.; The University of Edinburgh, Edinburgh, U.K.; and the University of 779 Portsmouth, Portsmouth, U.K. Since 1999, he has been with Electronics 780 and Computer Science, the University of Southampton, Southampton, 781 U.K., where he is currently a Professor of intelligent systems and signal 782 processing. He is also a Distinguished Adjunct Professor with King Abdulaziz 783 University, Jeddah, Saudi Arabia. He is the author of over 500 research 784 papers. His research interests include adaptive signal processing, wireless 785 communications, modeling and identification of nonlinear systems, neural 786 network and machine learning, intelligent control system design, and 787 evolutionary computation methods and optimization.

788 Dr. Chen is a Fellow of the Institution of Engineering and Technology and 789 an ISI highly cited researcher in engineering (March 2004). In 2014, he was 790 elected as a Fellow of the United Kingdom Royal Academy of Engineering.

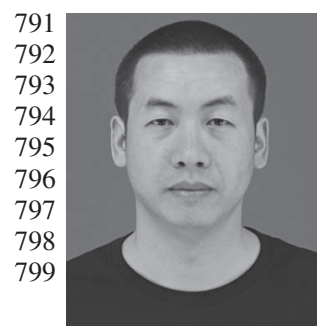

Liang Feng received the B.Eng. degree in applied physics from Dalian University of Technology, Dalian, China, in 2004. He is currently working toward the Master's degree with Nankai University, Nankai, China, in 2012.

From 2004 to 2012, he carried out electronic countermeasures research with the Luoyang Electronic Equipment Center, China. His research interests include cellular mobile communications.

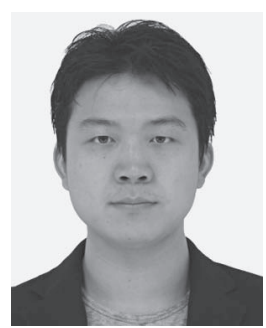

Yifeng Xie received the B.S. degree in electronic 800 and information engineering from Nanjing Forestry 801 University, Nanjing, China, in 2011 and the M.S. 802 degree in communication and information systems 803 from Nankai University, Nankai, China, in 2014. 804

His research interests include stochastic geometry 805 and heterogeneous networks. 806

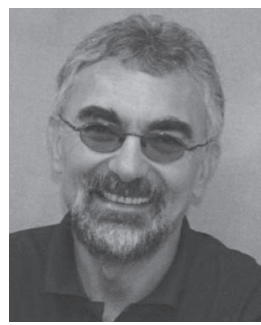

Lajos Hanzo (F'08) received the Master's and 807 D.Sc. degrees in electronics and the Doctor Honoris 808 Causa from the Technical University of Budapest, 809 Budapest, Hungary, in 1976 and 1983, respectively. 810

During his 38-year career in telecommunications, 811 he has held various research and academic posts in 812 Hungary, Germany, and the U.K. Since 1986, he has 813 been with the School of Electronics and Computer 814 Science, University of Southampton, Southampton, 815 U.K., where he is the Chair in telecommunications. 816 $\mathrm{He}$ has successfully supervised about 100 Ph.D. 817 students. He is the author or coauthor of 20 John Wiley/IEEE Press books 818 on mobile radio communications, totalling in excess of 10000 pages, and of 819 more than 1400 research entries on IEEE Xplore. Currently, he is directing a 820 100 -strong academic research team, working on a range of research projects 821 in the field of wireless multimedia communications sponsored by industry, 822 the Engineering and Physical Sciences Research Council (EPSRC) U.K., the 823 European Research Council's Advanced Fellow Grant, and the Royal Society's 824 Wolfson Research Merit Award. He is an enthusiastic supporter of industrial 825 and academic liaison, and he offers a range of industrial courses.

Dr. Hanzo has acted both as a Technical Program Committee Chair and as a 827 General Chair of IEEE conferences, has presented keynote lectures, and has 828 received a number of distinctions. He is a Governor of the IEEE Vehicular 829 Technology Society. From 2008 to 2012, he was the Editor-in-Chief of the IEEE 830 Press and a Chaired Professor with Tsinghua University, Beijing, China. His 831 research is funded by the European Research Council's Senior Research Fellow 832 Grant. He is a Fellow of the Royal Academy of Engineering, the Institution of 833 Engineering and Technology, and the European Association for Research and 834 Signal Processing. 


\section{AUTHOR QUERIES}

\section{AUTHOR PLEASE ANSWER ALL QUERIES}

AQ1 = Note that Ref. [7] was split into two. Consequently, the reference list and bibliographic citations were renumbered. Please check.

END OF ALL QUERIES 